\title{
Exponential Antisynchronization Control of Stochastic Memristive Neural Networks with Mixed Time-Varying Delays Based on Novel Delay-Dependent or Delay-Independent Adaptive Controller
}

\author{
Minghui Yu, ${ }^{1,2}$ Weiping Wang, ${ }^{1,2}$ Xiong Luo, ${ }^{1,2}$ Linlin Liu, ${ }^{3}$ and Manman Yuan ${ }^{1,2}$ \\ ${ }^{1}$ School of Computer and Communication Engineering, University of Science and Technology Beijing, \\ Beijing 100083, China \\ ${ }^{2}$ Beijing Key Laboratory of Knowledge Engineering for Materials Science, Beijing 100083, China \\ ${ }^{3}$ Institute of Microstructure and Properties of Advanced Materials, Beijing University of Technology, Beijing 100124, China
}

Correspondence should be addressed to Weiping Wang; shiya666888@126.com and Xiong Luo; xluo@ustb.edu.cn

Received 2 December 2016; Accepted 6 February 2017; Published 27 March 2017

Academic Editor: Honglei Xu

Copyright (C) 2017 Minghui Yu et al. This is an open access article distributed under the Creative Commons Attribution License, which permits unrestricted use, distribution, and reproduction in any medium, provided the original work is properly cited.

\begin{abstract}
The global exponential antisynchronization in mean square of memristive neural networks with stochastic perturbation and mixed time-varying delays is studied in this paper. Then, two kinds of novel delay-dependent and delay-independent adaptive controllers are designed. With the ability of adapting to environment changes, the proposed controllers can modify their behaviors to achieve the best performance. In particular, on the basis of the differential inclusions theory, inequality theory, and stochastic analysis techniques, several sufficient conditions are obtained to guarantee the exponential antisynchronization between the drive system and response system. Furthermore, two numerical simulation examples are provided to the validity of the derived criteria.
\end{abstract}

\section{Introduction}

Memristor is a nonlinear resistor with memory function. Because of the nonlinear nature, it has been found that memristor responds well to the variable strength of synapses in the human brain. Therefore, we use memristor instead of resistor to reform new neural networks model named memristive neural networks (MNNs). In recent decades, dynamic behaviors analysis of MNNs has been attracting increasing attentions [1-3].

As a typical dynamic behavior, the stability and synchronization problems of MNNs have been widely discussed, including exponential synchronization $[4,5]$, lag synchronization [6], finite time synchronization $[7,8]$, and antisynchronization $[9,10]$. In communication system, the digital signal is transmitted by switching back and forth continuously between synchronization and antisynchronization, which strengthen security and secrecy. Besides that, the antisynchronization analysis of MNNs can provide the designers with some amazing properties, extensive flexibility, and opportunities. Thus, there are a few articles dealing with the antisynchronization issues of MNNs [11-15]. In [11], they studied the exponential antisynchronization in mean square of MNNs with the uncertain terms which include nonmodeled dynamics with boundary and stochastic perturbations. By an intermittent sampled-data controller [12] and utilizing matrix measure approach and Halanay inequality [13], the antisynchronization control of MNNs with time-varying delays has been addressed. Wang et al. discussed the antisynchronization control of MNNs with multiple proportional delays [14] and mixed time-varying delays [15]. Recently, the antisynchronization control has been widely employed in many fields, for example, secure communication, harmonic oscillation generation, and information science [16-18].

In practice, because of certain switching speeds of the amplifiers, time delays are often encountered in hardware implementation [19], which may affect the stability of the system, even result in oscillation, divergence, and instability 
phenomena. Consequently, a great deal of effort has been devoted to the stability analysis of MNNs with various types of time delays [20-22]. Moreover, since an amount of parallel pathways of multiple axon sizes and lengths reside in the MNNs, such a unique nature can be appropriately modeled by distributed delay which means the signal transmission is distributed over a specific period of time. Hence, the authors in [23-25] have concentrated on the mixed delays. Meng and Xiang [24] considered a class of recurrent MNNs with mixed time-varying delays, in which the passivity and exponential passivity were investigated.

Furthermore, in [25], Yang et al. studied the $p$ th moment exponential stochastic synchronization for MNNs with discrete and distributed time-varying delays. However, the discrete delay $\tau(t)$ in [25] must be differentiable. Clearly, such a constraint on the delay term $\tau(t)$ was relatively strong. So, we discuss two kinds of discrete time-varying delay in this paper. One is that its derivative is bounded; the other is that it is not differentiable, or the derivative is unknown or unrestricted.

On the other hand, as a result of random fluctuations from the release of neurotransmitters or other probabilistic causes in the nervous system, synaptic transmission is indeed a process accompanied by noise. The stability analysis of MNNs with stochastic perturbation has aroused great interests of many researchers [26-28]. For example, Song and Wen [27] investigated the stochastic MNNs with mixed delays and proposed the exponential synchronization criteria in the pth moment. Subsequently, Bao et al. [28] discussed a class of coupled stochastic MNNs with probabilistic timevarying delay in order to achieve exponential synchronization. However, to the best of our knowledge, the research on the exponential antisynchronization analysis of stochastic MNNs with mixed delays is still an open problem.

Motivated by the above discussion, we focus our minds on the exponential antisynchronization problem of the MNNs with stochastic perturbation and mixed time-varying delays. Compared with other existing articles [11, 28, 29], our model is more complex and closer to the actual system; the obtained results are less conservative. The main contributions of this paper can be summarized in the following:

(1) The presented MNNs model contains not only stochastic perturbation but also two types of timevarying delays, namely, discrete and distributed timevarying delays.

(2) It is known that the time delays have a great influence on the stability of the MNNs, so the time delays cannot be neglected. As far as we know, many articles are based on the assumption that the time delay is bounded and derivable. Actually, it may happen that the time delay $\tau(t)$ is not differentiable or its derivative is unknown or unrestricted. Therefore, we study two kinds of discrete time-varying delay.

(3) Under two new types of controllers with delaydependent and delay-independent, the obtained criteria which need no excessive numerical calculation can be extended to other general MNNs models.
The rest structure of this paper is outlined as follows. In Section 2, the models of the stochastic MNNs with mixed time-varying delays and some preliminaries are introduced. In Section 3, the main results on exponential antisynchronization of the stochastic MNNs are derived. In Section 4, some numerical simulations are presented to demonstrate the efficiency of the theoretical results. In Section 5, the conclusion is showed.

\section{Model Description and Preliminaries}

On the basis of the above discussion, we propose a class of MNNs with discrete and distributed time-varying delays described by the following differential equations:

$$
\begin{aligned}
\frac{d x_{i}(t)}{d t}= & -d_{i} x_{i}(t)+\sum_{j=1}^{n} a_{i j}\left(x_{i}(t)\right) f_{j}\left(x_{j}(t)\right) \\
& +\sum_{j=1}^{n} b_{i j}\left(x_{i}(t-\tau(t))\right) g_{j}\left(x_{j}(t-\tau(t))\right) \\
& +\sum_{j=1}^{n} c_{i j}\left(x_{i}(t)\right) \int_{t-\rho(t)}^{t} f_{j}\left(x_{j}(s)\right) d s+I_{i},
\end{aligned}
$$

where $n$ corresponds to the number of neurons in system (1). $x_{i}(t)$ denotes the voltage of the capacitor $C_{i}$ at time $t$. The selfinhibition connection weight is $d_{i}>0$. The neuron activation functions $f_{j}(\cdot)$ and $g_{j}(\cdot)$ are continuous and bounded. $\tau(t)$ and $\rho(t)$ are two time-varying delays satisfying $0 \leq \tau(t) \leq \theta_{1}$, $0 \leq \rho(t) \leq \theta_{2},|\dot{\tau}(t)| \leq \delta<1\left(\theta_{1}, \theta_{2}\right.$, and $\delta$ are positive constants). $I_{i}(t)$ represents the continuous external inputs on the $i$ th neuron at time $t . a_{i j}\left(x_{i}(t)\right), b_{i j}\left(x_{i}(t-\tau(t))\right)$, and $c_{i j}\left(x_{i}(t)\right)$ are the memristive connection weights:

$$
\begin{aligned}
a_{i j}\left(x_{i}(t)\right) & =\frac{M_{i j}}{C_{i}} \times \operatorname{sign}_{i j}, \\
c_{i j}\left(x_{i}(t)\right) & =\frac{\bar{M}_{i j}}{C_{i}} \times \operatorname{sign}_{i j}, \\
b_{i j}\left(x_{i}(t-\tau(t))\right) & =\frac{\widetilde{M}_{i j}}{C_{i}} \times \operatorname{sign}_{i j}, \\
\operatorname{sign}_{i j} & = \begin{cases}1, & i \neq j, \\
-1, & i=j,\end{cases}
\end{aligned}
$$

in which $M_{i j}, \widetilde{M}_{i j}$, and $\bar{M}_{i j}$ denote the memductances of memristors $R_{i j}, \widetilde{R}_{i j}$, and $\bar{R}_{i j}$, respectively. In addition, $R_{i j}$ represents the memristor between the activation function $f_{i}\left(x_{i}(t)\right)$ and $x_{i}(t), \widetilde{R}_{i j}$ represents the memristor between the activation function $g_{i}\left(x_{i}(t-\tau(t))\right)$ and $x_{i}(t)$, and $\bar{R}_{i j}$ represents the memristor between the activation function $\int_{t-\rho(t)}^{t} f_{i}\left(x_{i}(s)\right) d s$ and $x_{i}(t)$. As far as we know, capacitor $C_{i}$ is constant while memductances of memristors $M_{i j}, \widetilde{M}_{i j}$, and $\bar{M}_{i j}$ respond to change in pinched hysteresis loops [30]. As 
a consequence, $a_{i j}\left(x_{i}(t)\right), b_{i j}\left(x_{i}(t-\tau(t))\right)$, and $c_{i j}\left(x_{i}(t)\right)$ will change with time. Based on the current-voltage characteristic of the memristor, we let

$$
\begin{aligned}
a_{i j}\left(x_{i}(t)\right) & = \begin{cases}\widehat{a}_{i j}, & \left|x_{i}(t)\right|>\Phi_{i}, \\
\check{a}_{i j}, & \left|x_{i}(t)\right| \leq \Phi_{i},\end{cases} \\
b_{i j}\left(x_{i}(t-\tau(t))\right) & = \begin{cases}\widehat{b}_{i j}, & \left|x_{i}(t-\tau(t))\right|>\Phi_{i}, \\
\check{b}_{i j}, & \left|x_{i}(t-\tau(t))\right| \leq \Phi_{i},\end{cases} \\
c_{i j}\left(x_{i}(t)\right) & = \begin{cases}\widehat{c}_{i j}, & \left|x_{i}(t)\right|>\Phi_{i}, \\
\check{c}_{i j}, & \left|x_{i}(t)\right| \leq \Phi_{i},\end{cases}
\end{aligned}
$$

where the switching jumps $\Phi_{i}>0$, for $i, j=1,2, \ldots, n$. Then $\widehat{a}_{i j}, \check{a}_{i j}, \widehat{b}_{i j}, \check{b}_{i j}, \widehat{c}_{i j}$, and $\check{c}_{i j}$ are known constants with respect to the memristance. Moreover, the initial condition of system (1) is given to be $x(s)=\phi(s), \phi(s) \in C\left([-\tau, 0], R^{n}\right)$.

Remark 1. According to the above explanation, $a_{i j}\left(x_{i}(t)\right)$, $b_{i j}\left(x_{i}(t-\tau(t))\right)$, and $c_{i j}\left(x_{i}(t)\right)$ in system (1) are changed as the state of the memristance is switching or the state has switched. Therefore, the MNNs is considered as a time-varying system with state-dependent switching. When $a_{i j}\left(x_{i}(t)\right), b_{i j}\left(x_{i}(t-\right.$ $\tau(t)))$, and $c_{i j}\left(x_{i}(t)\right)$ are constants, system (1) becomes the general recurrent neural networks.

Since $a_{i j}\left(x_{i}(t)\right), b_{i j}\left(x_{i}(t-\tau(t))\right)$, and $c_{i j}\left(x_{i}(t)\right)$ are discontinuous, in this paper, the solutions of all the following systems are illustrated in Filippov's sense. $[\cdot, \cdot]$ represents the interval. Set $\bar{a}_{i j}=\max \left\{\widehat{a}_{i j}, \check{a}_{i j}\right\}, \underline{a}_{i j}=\min \left\{\widehat{a}_{i j}, \check{a}_{i j}\right\}, \bar{b}_{i j}=$ $\max \left\{\widehat{b}_{i j}, \check{b}_{i j}\right\}, \underline{b}_{i j}=\min \left\{\widehat{b}_{i j}, \check{b}_{i j}\right\}, \bar{c}_{i j}=\max \left\{\widehat{c}_{i j}, \check{c}_{i j}\right\}$, and $\underline{c}_{i j}=$ $\min \left\{\widehat{c}_{i j}, \check{c}_{i j}\right\}$ for $i, j=1,2, \ldots, n \cdot \operatorname{co}[u, v]$ denotes closure of the convex hull generated by real numbers $u$ and $v$. Considering system (1), we define the following set-valued maps:

$$
\begin{aligned}
& \operatorname{co}\left(a_{i j}\left(x_{i}(t)\right)\right)= \begin{cases}\widehat{a}_{i j}, & \left|x_{i}(t)\right|>\Phi_{i}, \\
\operatorname{co}\left\{\widehat{a}_{i j}, \check{a}_{i j}\right\}, & \left|x_{i}(t)\right|=\Phi_{i}, \\
\check{a}_{i j}, & \left|x_{i}(t)\right|<\Phi_{i}\end{cases} \\
& \operatorname{co}\left(b_{i j}\left(x_{i}(t-\tau(t))\right)\right) \\
& =\left\{\begin{array}{cl}
\widehat{b}_{i j}, & \left|x_{i}(t-\tau(t))\right|>\Phi_{i}, \\
\operatorname{co}\left\{\widehat{b}_{i j}, \check{b}_{i j}\right\}, & \left|x_{i}(t-\tau(t))\right|=\Phi_{i}, \\
\check{b}_{i j}, & \left|x_{i}(t-\tau(t))\right|<\Phi_{i}
\end{array}\right. \\
& \operatorname{co}\left(c_{i j}\left(x_{i}(t)\right)\right)= \begin{cases}\widehat{c}_{i j}, & \left|x_{i}(t)\right|>\Phi_{i}, \\
\operatorname{co}\left\{\widehat{c}_{i j}, \check{c}_{i j}\right\}, & \left|x_{i}(t)\right|=\Phi_{i}, \\
\check{c}_{i j}, & \left|x_{i}(t)\right|<\Phi_{i} .\end{cases}
\end{aligned}
$$

Obviously, $\operatorname{co}\left\{\widehat{a}_{i j}, \check{a}_{i j}\right\}=\left[\underline{a}_{i j}, \bar{a}_{i j}\right], \operatorname{co}\left\{\widehat{b}_{i j}, \check{b}_{i j}\right\}=\left[\underline{b}_{i j}, \bar{b}_{i j}\right]$, and $\operatorname{co}\left\{\widehat{c}_{i j}, \check{c}_{i j}\right\}=\left[\underline{c}_{i j}, \bar{c}_{i j}\right]$, for $i, j=1,2, \ldots, n$. By the theory of differential inclusions, system (1) can be written as follows:

$$
\begin{aligned}
\frac{d x_{i}(t)}{d t} \in & -d_{i} x_{i}(t)+\sum_{j=1}^{n} \operatorname{co}\left(a_{i j}\left(x_{i}(t)\right)\right) f_{j}\left(x_{j}(t)\right) \\
& +\sum_{j=1}^{n} \operatorname{co}\left(b_{i j}\left(x_{i}(t-\tau(t))\right)\right) g_{j}\left(x_{j}(t-\tau(t))\right) \\
& +\sum_{j=1}^{n} \operatorname{co}\left(c_{i j}\left(x_{i}(t)\right)\right) \int_{t-\rho(t)}^{t} f_{j}\left(x_{j}(s)\right) d s+I_{i},
\end{aligned}
$$

or equivalently, for $i, j=1,2, \ldots, n$, there exist $\widehat{a}_{i j}\left(x_{i}(t)\right) \epsilon$ $\operatorname{co}\left(a_{i j}\left(x_{i}(t)\right)\right), \widehat{b}_{i j}\left(x_{i}(t-\tau(t))\right) \in \operatorname{co}\left(b_{i j}\left(x_{i}(t-\tau(t))\right)\right)$, and $\widehat{c}_{i j}\left(x_{i}(t)\right) \in \operatorname{co}\left(c_{i j}\left(x_{i}(t)\right)\right)$, such that

$$
\begin{aligned}
\frac{d x_{i}(t)}{d t}= & -d_{i} x_{i}(t)+\sum_{j=1}^{n} \widehat{a}_{i j}\left(x_{i}(t)\right) f_{j}\left(x_{j}(t)\right) \\
& +\sum_{j=1}^{n} \widehat{b}_{i j}\left(x_{i}(t-\tau(t))\right) g_{j}\left(x_{j}(t-\tau(t))\right) \\
& +\sum_{j=1}^{n} \widehat{c}_{i j}\left(x_{i}(t)\right) \int_{t-\rho(t)}^{t} f_{j}\left(x_{j}(s)\right) d s+I_{i} .
\end{aligned}
$$

In this paper, consider system (5) or (6) as the drive system; then the corresponding response system is designed by the following form:

$$
\begin{aligned}
& d y_{i}(t) \in\left\{-d_{i} y_{i}(t)+\sum_{j=1}^{n} \operatorname{co}\left(a_{i j}\left(x_{i}(t)\right)\right) f_{j}\left(y_{j}(t)\right)\right. \\
& +\sum_{j=1}^{n} \operatorname{co}\left(b_{i j}\left(x_{i}(t-\tau(t))\right)\right) g_{j}\left(y_{j}(t-\tau(t))\right) \\
& +\sum_{j=1}^{n} \operatorname{co}\left(c_{i j}\left(x_{i}(t)\right)\right) \int_{t-\rho(t)}^{t} f_{j}\left(y_{j}(s)\right) d s+I_{i} \\
& \left.+u_{i}(t)\right\} d t+\beta_{i}\left(t, x_{i}(t)+y_{i}(t), x_{i}(t-\tau(t))\right. \\
& \left.+y_{i}(t-\tau(t))\right) d \omega_{i}(t)
\end{aligned}
$$

or equivalently, for $i, j=1,2, \ldots, n$, there exist $\widehat{a}_{i j}\left(x_{i}(t)\right) \epsilon$ $\operatorname{co}\left(a_{i j}\left(x_{i}(t)\right)\right), \widehat{b}_{i j}\left(x_{i}(t-\tau(t))\right) \in \operatorname{co}\left(b_{i j}\left(x_{i}(t-\tau(t))\right)\right), \widehat{c}_{i j}\left(x_{i}(t)\right) \in$ $\operatorname{co}\left(c_{i j}\left(x_{i}(t)\right)\right)$, such that

$$
\begin{gathered}
d y_{i}(t)=\left\{-d_{i} y_{i}(t)+\sum_{j=1}^{n} \widehat{a}_{i j}\left(x_{i}(t)\right) f_{j}\left(y_{j}(t)\right)\right. \\
+\sum_{j=1}^{n} \widehat{b}_{i j}\left(x_{i}(t-\tau(t))\right) g_{j}\left(y_{j}(t-\tau(t))\right)
\end{gathered}
$$




$$
\begin{aligned}
& \left.+\sum_{j=1}^{n} \widehat{c}_{i j}\left(x_{i}(t)\right) \int_{t-\rho(t)}^{t} f_{j}\left(y_{j}(s)\right) d s+I_{i}+u_{i}(t)\right\} d t \\
& +\beta_{i}\left(t, x_{i}(t)+y_{i}(t), x_{i}(t-\tau(t))\right. \\
& \left.+y_{i}(t-\tau(t))\right) d \omega_{i}(t),
\end{aligned}
$$

where $u_{i}(t), i=1,2, \ldots, n$, are the appropriate adaptive controllers designed to achieve the exponential antisynchronization stability. $\beta_{i}\left(t, x_{i}(t)+y_{i}(t), x_{i}(t-\tau(t))+y_{i}(t-\right.$ $\tau(t))) d \omega_{i}(t)$ denotes the stochastic perturbation, where $\omega_{i}(t)$ is an $n$-dimensional Wiener process. It is defined on a complete probability space with a natural filtration $\{\mathscr{F}\}_{t \geq 0}$ (i.e., $\mathscr{F}_{t}=\beta\{\omega(s): 0 \leq s \leq t\}$ ).

Remark 2. Compared with the articles already published [28, 29], the proposed system contains not only discrete timevarying delay $\tau(t)$ but also distributed time-varying delay $\rho(t)$, while the stochastic perturbation is also taken into account. Therefore, the obtained results have a high value of practical application in this paper.

As a matter of convenience, we will take advantage of the following assumptions.

Assumption 3. For $\forall x, y \in R^{N}$, we assume the activation functions $f_{j}(\cdot)$ and $g_{j}(\cdot)$ are odd and satisfy the Lipschitz continuous condition with positive constants $L_{j}$ and $Q_{j}$, such that

$$
\begin{aligned}
& \left|f_{j}(x)-f_{j}(y)\right| \leq L_{j}|x-y|, \\
& \left|g_{j}(x)-g_{j}(y)\right| \leq Q_{j}|x-y|,
\end{aligned}
$$

$$
j=1,2, \ldots, n \text {. }
$$

Assumption 4. $\beta_{i}=: R_{+} \times R \times R \rightarrow R$ is locally Lipschitz continuous and meets the linear growth condition with $\beta_{i}(t, 0,0)=0$. There exist nonnegative constants $G_{i}, K_{i}$, such that, for $i, j=1,2, \ldots, n$,

$$
\begin{gathered}
\text { Trace }\left\{\left\|\beta_{i}\left(t, e_{i}(t), e_{i}(t-\tau(t))\right)\right\|^{2}\right\} \\
\leq G_{i}\left\|e_{i}(t)\right\|^{2}+K_{i}\left\|e_{i}(t-\tau(t))\right\|^{2} .
\end{gathered}
$$

Assumption 5. For $i, j=1, \ldots, n$,

$$
\begin{gathered}
\operatorname{co}\left[\underline{a}_{i j}, \bar{a}_{i j}\right] f_{j}\left(y_{j}(t)\right)+\operatorname{co}\left[\underline{a}_{i j}, \bar{a}_{i j}\right] f_{j}\left(x_{j}(t)\right) \\
\subseteq \operatorname{co}\left[\underline{a}_{i j}, \bar{a}_{i j}\right]\left(f_{j}\left(y_{j}(t)\right)+f_{j}\left(x_{j}(t)\right)\right), \\
\operatorname{co}\left[\underline{b}_{i j}, \bar{b}_{i j}\right] g_{j}\left(y_{j}(t-\tau(t))\right)+\operatorname{co}\left[\underline{b}_{i j}, \bar{b}_{i j}\right] \\
\cdot g_{j}\left(x_{j}(t-\tau(t))\right) \subseteq \operatorname{co}\left[\underline{b}_{i j}, \bar{b}_{i j}\right] \\
\cdot\left(g_{j}\left(y_{j}(t-\tau(t))\right)+g_{j}\left(x_{j}(t-\tau(t))\right)\right), \\
\operatorname{co}\left[\underline{c}_{i j}, \bar{c}_{i j}\right] f_{j}\left(y_{j}(t)\right)+\operatorname{co}\left[\underline{c}_{i j}, \bar{c}_{i j}\right] f_{j}\left(x_{j}(t)\right) \\
\subseteq \operatorname{co}\left[\underline{c}_{i j}, \bar{c}_{i j}\right]\left(f_{j}\left(y_{j}(t)\right)+f_{j}\left(x_{j}(t)\right)\right) .
\end{gathered}
$$

Remark 6. In [4], however, the error system was defined based on the following assumption:

$$
\begin{gathered}
\operatorname{co}\left[\underline{a}_{i j}, \bar{a}_{i j}\right] f_{j}\left(y_{j}(t)\right)-\operatorname{co}\left[\underline{a}_{i j}, \bar{a}_{i j}\right] f_{j}\left(x_{j}(t)\right) \\
\subseteq \operatorname{co}\left[\underline{a}_{i j}, \bar{a}_{i j}\right]\left(f_{j}\left(y_{j}(t)\right)-f_{j}\left(x_{j}(t)\right)\right) .
\end{gathered}
$$

In fact, this assumption has been proved not always to be correct in [5]. Recently, many researchers have tried to explore a novel and appropriate way to solve this problem. So far, there are two kinds of convincing method. One is, in [31], according to the switching parameter $\Phi_{i}$; the authors discussed the parameter in four cases and obtained several synchronization conditions of the chaotic MNNs with time delays. The other is, in [11, 15-17], by Assumption 5; the authors turned to study the antisynchronization issues of MNNs.

Now, we define the following error system:

$$
e_{i}(t)=y_{i}(t)+x_{i}(t), \quad i=1,2, \ldots, n .
$$

According to the theories of set-valued maps, stochastic differential inclusions, and Assumption 5, we derive the error dynamical system as follows:

$$
\begin{aligned}
& d e_{i}(t) \in\left\{-d_{i} e_{i}(t)+\sum_{j=1}^{n} \operatorname{co}\left(a_{i j}\left(x_{i}(t)\right)\right) h_{j}\left(e_{j}(t)\right)\right. \\
& +\sum_{j=1}^{n} \operatorname{co}\left(b_{i j}\left(x_{i}(t-\tau(t))\right)\right) z_{j}\left(e_{j}(t-\tau(t))\right) \\
& +\sum_{j=1}^{n} \operatorname{co}\left(c_{i j}\left(x_{i}(t)\right)\right) \int_{t-\rho(t)}^{t} h_{j}\left(e_{j}(s)\right) d s+2 I_{i} \\
& \left.+u_{i}(t)\right\} d t+\beta_{i}\left(t, e_{i}(t), e_{i}(t-\tau(t))\right) d \omega_{i}(t),
\end{aligned}
$$

or equivalently, for $i, j=1,2, \ldots, n$, there exist $\tilde{a}_{i j}\left(x_{i}(t)\right) \epsilon$ $\operatorname{co}\left(a_{i j}\left(x_{i}(t)\right)\right), \widetilde{b}_{i j}\left(x_{i}(t-\tau(t))\right) \in \operatorname{co}\left(b_{i j}\left(x_{i}(t-\tau(t))\right)\right)$, and $\widetilde{c}_{i j}\left(x_{i}(t)\right) \in \operatorname{co}\left(c_{i j}\left(x_{i}(t)\right)\right)$, such that

$$
\begin{aligned}
& d e_{i}(t)=\left\{-d_{i} e_{i}(t)+\sum_{j=1}^{n} \widetilde{a}_{i j}\left(x_{i}(t)\right) h_{j}\left(e_{j}(t)\right)\right. \\
& +\sum_{j=1}^{n} \widetilde{b}_{i j}\left(x_{i}(t-\tau(t))\right) z_{j}\left(e_{j}(t-\tau(t))\right) \\
& +\sum_{j=1}^{n} \widetilde{c}_{i j}\left(x_{i}(t)\right) \int_{t-\rho(t)}^{t} h_{j}\left(e_{j}(s)\right) d s+2 I_{i} \\
& \left.+u_{i}(t)\right\} d t+\beta_{i}\left(t, e_{i}(t), e_{i}(t-\tau(t))\right) d \omega_{i}(t),
\end{aligned}
$$

where $h_{j}\left(e_{j}(t)\right)=f_{j}\left(y_{j}(t)\right)+f_{j}\left(x_{j}(t)\right), z_{j}\left(e_{j}(t-\tau(t))\right)=$ $g_{j}\left(y_{j}(t-\tau(t))\right)+g_{j}\left(x_{j}(t-\tau(t))\right)$, and $h_{j}\left(e_{j}(s)\right)=f_{j}\left(y_{j}(s)\right)+$ $f_{j}\left(x_{j}(s)\right)$. 
Since the activation functions $f_{i}(\cdot)$ and $g_{i}(\cdot)$ are odd and bounded, in the light of the Assumption 3, for $\forall x, y \in R$, it is so clear that

$$
\begin{aligned}
& \left|f_{j}(x)+f_{j}(y)\right| \leq L_{j}|x+y|, \\
& \left|g_{j}(x)+g_{j}(y)\right| \leq Q_{j}|x+y| .
\end{aligned}
$$

According to the definition of functions $h_{j}(\cdot)$ and $z_{j}(\cdot)$, we deduce

$$
\begin{gathered}
\left|h_{j}\left(e_{j}(t)\right)\right| \leq L_{j}\left|e_{j}(t)\right| \\
\left|z_{j}\left(e_{j}(t-\tau(t))\right)\right| \leq Q_{j}\left|e_{j}(t-\tau(t))\right| .
\end{gathered}
$$

Next, we introduce a definition and some lemmas as preparation.

Lemma 7. For the stochastic system,

$$
\begin{aligned}
d e(t)= & g(t, e(t), e(t-\tau(t))) d t \\
& +\beta(t, e(t), e(t-\tau(t))) d \omega(t),
\end{aligned}
$$

where $\omega(t)$ is the Wiener process and it is obvious that $E \omega(t)=$ $0 . \mathscr{L V}(t, e(t))$ is the operator defined as follows:

$$
\begin{aligned}
& \mathscr{L} V(t, e(t))=V_{t}(t, e(t))+V_{e}(t, e(t)) g(t, e(t), e(t \\
& -\tau(t)))+\frac{1}{2} \operatorname{Trace}\left[\beta^{T}(t, e(t), e(t-\tau(t)))\right. \\
& \left.\quad \times V_{e e} \beta(t, e(t), e(t-\tau(t)))\right]
\end{aligned}
$$

where

$$
\begin{aligned}
& V_{t}(t, e(t))=\frac{\partial V(t, e(t))}{\partial t} \\
& V_{e}(t, e(t)) \\
& \quad=\left(\frac{\partial V(t, e(t))}{\partial x_{1}}, \frac{\partial V(t, e(t))}{\partial x_{2}}, \ldots, \frac{\partial V(t, e(t))}{\partial x_{n}}\right), \\
& V_{e e}=\left(\frac{\partial^{2} V(t, e(t))}{\partial x_{i} \partial x_{j}}\right)_{n \times n} .
\end{aligned}
$$

Lemma 8 (see [32]). If $X$ and $Y$ are real matrices which have appropriate dimensions, then there exists a constant $\varepsilon>0$, such that

$$
X^{T} Y+Y^{T} X \leq \varepsilon X^{T} X+\frac{1}{\varepsilon} Y^{T} Y .
$$

Lemma 9 (see [33], Jensen's inequality). For any constant matrix $M>0$, a scalar $a \leq b$, and a vector function $e(s):[a, b] \rightarrow R^{n}$, then the following inequality holds:

$$
\begin{aligned}
& {\left[\int_{a}^{b} e(s) d s\right]^{T} M\left[\int_{a}^{b} e(s) d s\right]} \\
& \quad \leq(b-a)\left[\int_{a}^{b} e^{T}(s) M e(s) d s\right] .
\end{aligned}
$$

Definition 10. The error system $e(t)$ with mixed delays and stochastic perturbations can exponentially converge to zero in mean square if there exist positive constants $\mu>0$ and $\nu>$ 0 , such that $E\left\{\|e(t)\|^{2}\right\} \leq \mu e^{(-v t)}$, for $t \geq 0$, where $v$ is called the exponential convergence rate. Systems (6) and (8) are said to be exponential antisynchronization in mean square sense.

\section{Main Results}

In this section, we get some sufficient criteria to achieve the exponential antisynchronization of the stochastic MNNs with mixed time-varying delays. Then two corollaries are also derived for the stochastic MNNs. The novel delay-dependent adaptive controller $u_{i}(t)$ is designed as follows:

$$
\begin{aligned}
& u_{i}(t)=-k_{i}(t) e_{i}(t)-m_{i}(t) e_{i}(t-\tau(t))-2 I_{i}, \\
& \dot{k}_{i}(t)=p_{i}\left\|e_{i}(t)\right\|^{2} e^{\nu t}, \quad k_{i}(0)=0, \\
& \dot{m}_{i}(t)=q_{i}\left\|e_{i}(t-\tau(t)) e_{i}(t)\right\| e^{\nu t}, \quad m_{i}(0)=0,
\end{aligned}
$$

where the constants $p_{i}>0, q_{i}>0$, for $i=1,2, \ldots, n$.

For convenience, some notations are given. Let $\stackrel{\circ}{a}_{i j}=$ $\max \left\{\left|\widehat{a}_{i j}\right|,\left|\check{a}_{i j}\right|\right\}, \stackrel{\circ}{i j}_{i j}=\max \left\{\left|\widehat{b}_{i j}\right|,\left|\check{b}_{i j}\right|\right\}$, and $\stackrel{\circ}{c}_{i j}=\max \left\{\left|\widehat{c}_{i j}\right|,\left|\check{c}_{i j}\right|\right\}$, for $i, j=1,2, \ldots, n$.

Theorem 11. Under Assumptions 3, 4, 5, and 13, systems (6) and (8) achieve exponential antisynchronization in mean square sense under the delay-dependent adaptive controller (23). For a given constant $\nu>0$, if there exist constants $r_{i}>0$, $s_{i}>0$ such that the conditions hold

$$
\begin{aligned}
r_{i} & =\frac{1}{2}\left\{\nu-2 d_{i}+n+G_{i}+\frac{n e^{\nu \theta_{1}}}{1-\delta}+n \theta_{2} \frac{e^{\nu \theta_{2}}-1}{v}\right. \\
& \left.+\sum_{j=1}^{n}\left[\stackrel{\circ}{a}_{i j}^{2} L_{j}^{2}+\stackrel{\circ}{b}_{i j}^{2} Q_{j}^{2}+{\stackrel{\circ}{c_{i j}}}^{2} L_{j}^{2}\right]\right\}, \\
s_{i} & =\sqrt{K_{i}},
\end{aligned}
$$

then error system (15) can be exponentially converged to zero.

Proof. We choose the following Lyapunov functional:

$$
V(t, e(t))=\sum_{k=1}^{4} V_{k}(t,(e(t))),
$$

where

$$
\begin{aligned}
& V_{1}(t, e(t))=\sum_{i=1}^{n} e^{v t} e_{i}^{2}(t) \\
& V_{2}(t, e(t))=\sum_{i=1}^{n} \frac{\left(k_{i}(t)-r_{i}\right)^{2}}{p_{i}}+\sum_{i=1}^{n} \frac{\left(m_{i}(t)-s_{i}\right)^{2}}{q_{i}}
\end{aligned}
$$




$$
\begin{aligned}
& V_{3}(t, e(t))=\sum_{i=1}^{n} \frac{1}{(1-\delta)} \sum_{j=1}^{n} \int_{t-\tau(t)}^{t} e_{j}^{2}(s) e^{\nu\left(s+\theta_{1}\right)} d s, \\
& V_{4}(t, e(t))=\theta_{2} \sum_{i=1}^{n} \sum_{j=1}^{n} \int_{-\theta_{2}}^{0} \int_{t+s}^{t} e_{j}^{2}(z) e^{\gamma(z-s)} d z d s .
\end{aligned}
$$

Then we have

$$
V(t, e(t)) \geq e^{\vartheta t}\|e(t)\|^{2} .
$$

By Itô differential formula, we obtain the stochastic derivative of $V(t)$ in the form of

$$
\begin{aligned}
d V(t, e(t))= & \sum_{i=1}^{4} \mathscr{L} V_{i}(t, e(t)) d t+2 e^{\vartheta t} \\
& \times \sum_{i=1}^{n} \beta_{i}\left(t, e_{i}(t), e_{i}(t-\tau(t))\right) d \omega_{i}(t) .
\end{aligned}
$$

From Lemma 7, it is clear that

$$
\begin{aligned}
& \mathscr{L}_{1}(t, e(t))=\sum_{i=1}^{n} \nu e^{\nu t} e_{i}^{2}(t)+2 \sum_{i=1}^{n} e^{\nu t} e_{i}(t)\left[-d_{i} e_{i}(t)\right. \\
& +\sum_{j=1}^{n} \widetilde{a}_{i j}\left(x_{i}(t)\right) h_{j}\left(e_{j}(t)\right) \\
& +\sum_{j=1}^{n} \widetilde{b}_{i j}\left(x_{i}(-\tau(t))\right) z_{j}\left(e_{j}(t-\tau(t))\right) \\
& +\sum_{j=1}^{n} \widetilde{c}_{i j}\left(x_{i}(t)\right) \int_{t-\rho(t)}^{t} h_{j}\left(e_{j}(s)\right) d s-k_{i}(t) e_{i}(t) \\
& \left.-m_{i}(t) e_{i}(t-\tau(t))\right] \\
& +e^{\nu t} \sum_{i=1}^{n} \operatorname{Trace}\left[\beta_{i}\left(t, e_{i}(t), e_{i}(t-\tau(t))\right)^{T}\right. \\
& \left.\quad \times \beta_{i}\left(t, e_{i}(t), e_{i}(t-\tau(t))\right)\right] \\
& \quad V_{2}(t, e(t))=2 e^{\nu t} \sum_{i=1}^{n}\left(k_{i}(t)-r_{i}\right) e_{i}^{2}(t) \\
& +2 e^{\nu t} \sum_{i=1}^{n}\left(m_{i}(t)-s_{i}\right) e_{i}(t-\tau(t)) e_{i}(t), \\
& \mathscr{L} V_{3}(t, e(t))=\sum_{i=1}^{n} \frac{1}{1-\delta} e_{j}^{\nu}(t-\tau(t)) e^{\nu\left(t+\theta_{1}\right)} \sum_{j=1}^{n} e_{j}^{2}(t)-\sum_{i=1}^{n} \frac{1}{1-\delta}(1) \leq e^{\nu t} \sum_{i=1}^{n} \frac{e^{\nu \theta_{1}}}{1-\delta}
\end{aligned}
$$

$$
\begin{aligned}
& \cdot \sum_{j=1}^{n} e_{j}^{2}(t)-e^{\nu t} \sum_{i=1}^{n} \sum_{j=1}^{n} e_{j}^{2}(t-\tau(t))=e^{\nu t} \frac{n e^{\nu \theta_{1}}}{1-\delta} \\
& \cdot \sum_{i=1}^{n} e_{i}^{2}(t)-n e^{\nu t} \sum_{i=1}^{n} e_{i}^{2}(t-\tau(t)), \\
& \mathscr{L} V_{4}(t, e(t)) \\
& =\theta_{2} e^{\nu t} \sum_{i=1}^{n} \sum_{j=1}^{n} \int_{-\theta_{2}}^{0}\left[e_{j}^{2}(t) e^{-\nu s}-e_{j}^{2}(t+s)\right] d s \\
& =\left.\theta_{2} \sum_{i=1}^{n} \sum_{j=1}^{n} e^{\nu t} e_{j}^{2}(t) \frac{e^{-\nu s}}{-\nu}\right|_{-\theta_{2}} ^{0} \\
& -\theta_{2} \sum_{i=1}^{n} \sum_{j=1}^{n} e^{\nu t} \int_{-\theta_{2}}^{0} e_{j}^{2}(t+s) d s \\
& \leq n e^{\nu t} \theta_{2} \sum_{i=1}^{n} \frac{e^{\nu \theta_{2}}-1}{\nu} e_{i}^{2}(t) \\
& -n \theta_{2} \sum_{i=1}^{n} e^{\nu t} \int_{t-\rho(t)}^{t} e_{i}^{2}(s) d s .
\end{aligned}
$$

Moreover, from mean value inequality and Assumption 3, we have

$$
\begin{aligned}
& 2 \sum_{i=1}^{n} e_{i}(t) \sum_{j=1}^{n} \widetilde{a}_{i j}\left(x_{i}(t)\right) h_{j}\left(e_{j}(t)\right) \\
& \quad \leq 2 \sum_{i=1}^{n} \sum_{j=1}^{n} e_{i}(t) \widetilde{a}_{i j}\left(x_{i}(t)\right) L_{j} e_{j}(t) \\
& \quad=\sum_{i=1}^{n} \sum_{j=1}^{n} \dot{a}_{i j}^{2} L_{j}^{2} e_{i}^{2}(t)+n \sum_{i=1}^{n} e_{i}^{2}(t) \\
& 2 \sum_{i=1}^{n} e_{i}(t) \sum_{j=1}^{n} \widetilde{b}_{i j}\left(x_{i}(t-\tau(t))\right) z_{j}\left(e_{j}(t-\tau(t))\right) \\
& \quad \leq 2 \sum_{i=1}^{n} \sum_{j=1}^{n} e_{i}(t) \widetilde{b}_{i j}\left(x_{i}(t-\tau(t))\right) Q_{j} e_{j}(t-\tau(t)) \\
& \quad=\sum_{i=1}^{n} \sum_{j=1}^{n} \dot{b}_{i j}^{2} Q_{j}^{2} e_{i}^{2}(t)+n \sum_{i=1}^{n} e_{i}^{2}(t-\tau(t)) \\
& 2 \sum_{i=1}^{n} s_{i} e_{i}(t) e_{i}(t-\tau(t)) \leq \sum_{i=1}^{n} e_{i}^{2}(t)+\sum_{i=1}^{n} s_{i}^{2} e_{i}^{2}(t-\tau(t)), \\
& 2 \sum_{i=1}^{n} e_{i}(t) \sum_{j=1}^{n} \widetilde{c}_{i j}\left(x_{i}(t)\right) \int_{t-\rho(t)}^{t} h_{j}\left(e_{j}(s)\right) d s \\
& \quad \leq 2 \sum_{i=1}^{n} \sum_{j=1}^{n} e_{i}(t) \widetilde{c}_{i j}\left(x_{i}(t)\right) L_{j} \int_{t-\rho(t)}^{t} e_{j}(s) d s \\
& \quad \leq \sum_{i=1}^{n} \sum_{j=1}^{n}\left[\dot{c}_{i j}^{2} L_{j}^{2} e_{i}^{2}(t)+\left(\int_{t-\rho(t)}^{t} e_{j}(s) d s\right)^{2}\right] \\
&
\end{aligned}
$$


and together with Lemma 9, it follows that

$$
\left(\int_{t-\rho(t)}^{t} e_{j}(s) d s\right)^{2} \leq \theta_{2} \int_{t-\rho(t)}^{t} e_{j}^{2}(s) d s .
$$

Then we get

$$
\begin{aligned}
& 2 \sum_{i=1}^{n} e_{i}(t) \sum_{j=1}^{n} \widetilde{c}_{i j}\left(x_{i}(t)\right) \int_{t-\rho(t)}^{t} h_{j}\left(e_{j}(s)\right) d s \\
& \quad \leq \sum_{i=1}^{n} \sum_{j=1}^{n} \dot{c}_{i j}^{2} L_{j}^{2} e_{i}^{2}(t)+n \theta_{2} \sum_{i=1}^{n} \int_{t-\rho(t)}^{t} e_{i}^{2}(s) d s .
\end{aligned}
$$

Under Assumption 4, one has

$$
\begin{aligned}
& \mathscr{L} V(t, e(t)) \leq e^{\nu t} \sum_{i=1}^{n}\left[\nu-2 d_{i}+n+G_{i}-2 r_{i}+\frac{n e^{\nu \theta_{1}}}{1-\delta}\right. \\
& \left.+n \theta_{2} \frac{e^{\nu \theta_{2}}-1}{v}+\sum_{j=1}^{n}\left(\stackrel{\circ}{a}_{i j}^{2} L_{j}^{2}+\stackrel{\circ}{b}_{i j}^{2} Q_{j}^{2}+\stackrel{\circ}{c}_{i j}^{2} L_{j}^{2}\right)\right] e_{i}^{2}(t) \\
& +e^{v t} \sum_{i=1}^{n} K_{i} e_{i}^{2}(t-\tau(t))+n e^{v t} \sum_{i=1}^{n} \theta_{2} \int_{t-\rho(t)}^{t} e_{i}^{2}(s) d s \\
& -e^{\nu t} \sum_{i=1}^{n} 2 s_{i} e_{i}(t) e_{i}(t-\tau(t)) \\
& -n \theta_{2} \sum_{i=1}^{n} e^{\nu t} \int_{t-\rho(t)}^{t} e_{i}^{2}(s) d s
\end{aligned}
$$

then we obtain the following estimation:

$$
\begin{aligned}
& \mathscr{L} V(t, e(t)) \leq e^{\nu t} \sum_{i=1}^{n}\left\{\left(\nu-2 d_{i}+n+G_{i}-2 r_{i}-1\right.\right. \\
& \left.+\frac{n e^{\nu \theta_{1}}}{1-\delta}+n \theta_{2} \frac{e^{\nu \theta_{2}}-1}{\nu}\right) \\
& \left.+\sum_{j=1}^{n}\left[\stackrel{\circ}{a}_{i j}^{2} L_{j}^{2}+\stackrel{\circ}{b}_{i j}^{2} Q_{j}^{2}+\stackrel{\circ}{c}_{i j}^{2} L_{j}^{2}\right]\right\} e_{i}^{2}(t)+e^{\nu t} \sum_{i=1}^{n}\left[K_{i}\right. \\
& \left.\quad-s_{i}^{2}\right] e_{i}^{2}(t-\tau(t)) .
\end{aligned}
$$

Let

$$
\begin{aligned}
r_{i}= & \frac{1}{2}\left\{\nu-2 d_{i}+n+G_{i}+\frac{n e^{\nu \theta_{1}}}{1-\delta}+n \theta_{2} \frac{e^{\nu \theta_{2}}-1}{v}\right. \\
& \left.+\sum_{j=1}^{n}\left[\stackrel{\circ}{a}_{i j}^{2} L_{j}^{2}+\stackrel{\circ}{b}_{i j}^{2} Q_{j}^{2}+\stackrel{\circ}{c}_{i j}^{2} L_{j}^{2}\right]\right\}, \\
s_{i} & =\sqrt{K_{i}},
\end{aligned}
$$

$$
i=1,2, \ldots, n \text {; }
$$

considering the condition of Theorem 11, we get

$$
\mathscr{L} V(t, e(t)) \leq-e^{v t}\left\|e(t)^{2}\right\| \leq 0 .
$$

It is easy to derive that

$$
E\{V(t, e(t))\} \leq E\{V(0, e(0))\}, \quad \forall t \geq 0 .
$$

Combining with (27), we finally have that

$$
\begin{aligned}
E\left\{\left\|e(t)^{2}\right\|\right\} & \leq E\{V(t, e(t))\} e^{-v t} \\
& \leq E\{V(0, e(0))\} e^{-v t} .
\end{aligned}
$$

Therefore, by Definition 10, we see that systems (6) and (8) can be exponentially synchronized in mean square sense with the exponential convergence rate $\nu$. The proof of Theorem 11 is completed.

Remark 12. In Theorem 11, we assumed that the delay $\tau(t)$ satisfies $0 \leq \dot{\tau}(t) \leq \delta$. Actually, when $\tau(t)$ is not differentiable or its derivatives are unknown or no bounded, Theorem 11 cannot be applicable any more. If the value of $\delta$ is unknown or not restriction, we will obtain the following corollary.

Assumption 13. The activation functions $f_{i}(\cdot)$ and $g_{i}(\cdot)$ are bounded; namely, there exist constants $\kappa_{i}, \omega_{i}$ such that

$$
\begin{aligned}
& \left|f_{i}(x)\right| \leq \kappa_{i}, \\
& \left|g_{i}(x)\right| \leq \omega_{i},
\end{aligned}
$$

$\forall x \in R, i=1,2, \ldots, n$.

It is obvious that

$$
\begin{aligned}
&\left|h_{j}\left(e_{j}(t)\right)\right| \leq 2 \kappa_{j}, \\
&\left|\int_{t-\rho(t)}^{t} h_{j}\left(e_{j}(s)\right) d s\right| \leq 2 \omega_{j} \theta_{2}, \\
& \quad j=1,2, \ldots, n .
\end{aligned}
$$

Corollary 14. If the time-varying delay $\tau(t)$ is nondifferentiable, or the value of $\dot{\tau}(t)$ is unknown or even the derivative $\dot{\tau}(t)$ is unrestricted. According to Assumptions 3, 4, and 13, system (15) will converge to zero. For a given constant $0<v<2 d_{i}-G_{i}$, if there exist constants $r_{i}>0, s_{i}>0$ and the conditions hold

$$
\begin{aligned}
r_{i}=\sum_{j=1}^{n} 2 \kappa_{j}\left(\stackrel{\circ}{a}_{i j}+\stackrel{\circ}{b}_{i j}\right)+\sum_{j=1}^{n} 2 \omega_{j} \theta_{2} \stackrel{\circ}{c}_{i j}, & \\
s_{i}=\sqrt{K_{i}}, \quad & i=1,2, \ldots, n,
\end{aligned}
$$

systems (6) and (8) are exponentially antisynchronized in the mean square sense under the following controller:

$$
\begin{aligned}
& u_{i}(t)=-k_{i}(t)-m_{i}(t) e_{i}(t-\tau(t))-2 I_{i}, \\
& \dot{k}_{i}(t)=p_{i}\left\|e_{i}(t)\right\| e^{\nu t}, \quad k_{i}(0)=0, \\
& \dot{m}_{i}(t)=q_{i}\left\|e_{i}(t-\tau(t)) e_{i}(t)\right\| e^{\nu t}, \quad m_{i}(0)=0 .
\end{aligned}
$$


Proof. We consider the following Lyapunov function:

$$
\begin{aligned}
V(t, e(t))= & \sum_{i=1}^{n} e^{\nu t} e_{i}^{2}(t)+\sum_{i=1}^{n} \frac{\left(k_{i}(t)-r_{i}\right)^{2}}{p_{i}} \\
& +\sum_{i=1}^{n} \frac{\left(m_{i}(t)-s_{i}\right)^{2}}{q_{i}} .
\end{aligned}
$$

Combining with Assumption 13, the proof of Corollary 14 is analogous to Theorem 11, so we omit it here.

Remark 15. Based on the assumption that the delay $\tau(t)$ is bounded and differential, Theorem 11 provides a delaydependent adaptive controller. It is worth mentioning that the criteria need no excessive numerical calculation such as solving LMIs [11] or computing complex algebraic conditions [28]. In Corollary 14, the delay $\tau(t)$ is not required to be differential but only bounded which makes our result more general. Further, there is an upper limit $\left(2 d_{i}-G_{i}\right)$ of the exponential convergence rate $v$. When the exponent rate $v$ exceeds the upper limit, system (15) may not be able to converge or even cause unpredictable result.

Let

$$
e(t)=\left(e_{1}(t), e_{2}(t), \ldots, e_{n}(t)\right)^{T} .
$$

The vector form of system (15) is given by

$$
\begin{aligned}
& d e(t)=[-D e(t)+A(x(t)) h(e(t)) \\
& +B(x(t-\tau(t))) \times z(e(t-\tau(t))) \\
& \left.+C(x(t)) \int_{t-\rho}^{t} h(e(s)) d s+2 I+u(t)\right] d t \\
& +\beta(t, e(t), e(-\tau(t))) d \omega(t),
\end{aligned}
$$

where $D=\operatorname{diag}\left(d_{1}, \ldots, d_{n}\right), A(x(t))=\left(\tilde{a}_{i j}\left(x_{i}(t)\right)\right)_{n \times n}, B(x(t-$ $\tau(t)))=\left(\widetilde{b}_{i j}\left(x_{i}(t-\tau(t))\right)\right)_{n \times n}, I=\left(I_{1}, \ldots, I_{n}\right), C(x(t))=$ $\left(\widetilde{c}_{i j}\left(x_{i}(t)\right)\right)_{n \times n}$, and $\omega(t)=\left(\omega_{1}(t), \ldots, \omega_{n}(t)\right)$. lemmas.

Moreover, we impose the following assumption and some

Assumption 16. If there exist appropriate positive constant matrices $T_{1}>0$ and $T_{2}>0$, such that the inequality holds

$$
\begin{aligned}
& \text { Trace }\left\{[\beta(t, e(t), e(t-\tau(t)))]^{T}\right. \\
& \quad \cdot[\beta(t, e(t), e(t-\tau(t)))]\} \leq e^{T}(t) T_{1}^{T} T_{1} e(t) \\
& \quad+e^{T}(t-\tau(t)) T_{2}^{T} T_{2} e(t-\tau(t)),
\end{aligned}
$$

for any $e(t), e(t-\tau(t)) \in R^{n}$ and $t \in R^{+}$.
Lemma 17 (see [24]). For any given nonnegative matrix $N \in$ $R^{n \times n}$, if there exist a constant $\nu>0$ and a vector function $x:[a, b] \rightarrow R^{n}$, the following differential inequality holds:

$$
\begin{aligned}
& \int_{b}^{a} e^{\nu s} e^{T}(s) N e(s) d s \\
& \quad \geq k\left(\int_{b}^{a} e(s) d s\right)^{T} N\left(\int_{b}^{a} e(s) d s\right),
\end{aligned}
$$

where $k=v /\left(e^{-v a}-e^{-v b}\right)$.

If there is no time delay item, the delay-independent adaptive controller can be described as follows:

$$
\begin{aligned}
u(t) & =-M(t) e(t)-2 I, \\
\dot{M}(t) & =\mu e^{\nu t} e^{T}(t) e(t)
\end{aligned}
$$

Next, we will discuss the antisynchronization of systems (6) and (8) under the above delay-independent adaptive controller $u(t)$.

Theorem 18. Under Assumption 13, for a given constant $\nu>0$, if there exist constant $m>0$ and matrixes $\Pi>0, \Xi>0$, the conditions hold as follows:

$$
\begin{aligned}
\Pi= & e^{\nu \theta_{1}}\left[\Delta_{2}^{-1} \Theta^{T} \Theta+T_{2}^{T} T_{2}\right], \\
\Xi & =\frac{e^{\nu \theta_{2}}-1}{\nu \Delta_{3} e^{\nu t}}, \\
m & =\frac{1}{2}\left(\nu-2 D+\Delta_{1} \stackrel{\circ}{ } \stackrel{\circ}{T}^{T}+\Delta_{1}^{-1} \Lambda^{T} \Lambda+\Delta_{2} \stackrel{\circ}{B} \dot{B}\right. \\
& \left.+\Delta_{3} \stackrel{\circ}{C}^{T}+\frac{\Pi}{1-\delta}+T_{1}^{T} T_{1}+\theta_{2} \Xi \Lambda^{T} \Lambda+1\right),
\end{aligned}
$$

where $\Lambda=\operatorname{diag}\left(L_{1}, L_{2}, \ldots, L_{n}\right), \Theta=\operatorname{diag}\left(Q_{1}, Q_{2}, \ldots, Q_{n}\right)$, $\stackrel{\circ}{A}=\left(\stackrel{\circ}{a}_{i j}\right)_{n \times n}, \stackrel{\circ}{B}=\left(\stackrel{\circ}{b}_{i j}\right)_{n \times n}$, and $\stackrel{\circ}{C}=\left(\stackrel{\circ}{c}_{i j}\right)_{n \times n}$. System (45) will converge to zero with the action of the above delay-independent adaptive controller.

Proof. We construct the following Lyapunov function:

$$
V(t, e(t))=\sum_{k=1}^{4} V_{k}(t, e(t)),
$$

where

$$
\begin{aligned}
& V_{1}(t, e(t))=e^{\nu t} e^{T}(t) e(t), \\
& V_{2}(t, e(t))=\frac{1}{\mu}(M(t)-m)^{2}, \\
& V_{3}(t, e(t))=\frac{\Pi}{(1-\delta)} \int_{t-\tau(t)}^{t} e^{\nu s} e^{T}(s) e(s) d s, \\
& V_{4}(t, e(t))=\Xi \int_{-\theta_{2}}^{0} \int_{t+s}^{t} e^{\nu z} h^{T}(e(z)) h(e(z)) d z d s .
\end{aligned}
$$


Then we have

$$
V(t, e(t)) \geq e^{\nu t} e^{T}(t) e(t) .
$$

According to Lemma 7, we get that

$$
\begin{aligned}
& \mathscr{L} V_{1}(t, e(t))=v e^{\nu t} e^{T}(t) e(t)+2 e^{\nu t} e^{T}(t)[-D e(t) \\
& +A(x(t)) h(e(t))+B(x(t-\tau(t))) \\
& \cdot z(e(t-\tau(t)))+C(x(t)) \int_{t-\rho(t)}^{t} h(e(s)) d s \\
& -M(t) e(t)]+e^{\nu t} \\
& \text { - Trace }\left[\beta^{T}(t, e(t), e(t-\tau(t)))\right. \\
& \times \beta(t, e(t), e(t-\tau(t)))], \\
& \mathscr{L} V_{2}(t, e(t))=\frac{2}{\mu}(M(t)-m) \dot{M}(t)=2(M(t)-m) \\
& \cdot e^{v t} e^{T}(t) e(t) \\
& \mathscr{L} V_{3}(t, e(t))=\frac{\Pi e^{\nu t}}{1-\delta} e^{T}(t) e(t)-\Pi \frac{e^{\nu(t-\tau(t))}}{1-\delta}(1 \\
& -\dot{\tau}(t)) e^{T}(t-\tau(t)) e(t-\tau(t)) \leq \frac{\Pi e^{\nu t}}{1-\delta} e^{T}(t) e(t) \\
& -\frac{\prod e^{\nu t}}{e^{\nu \theta_{1}}} e^{T}(t-\tau(t)) e(t-\tau(t)), \\
& \mathscr{L} V_{4}(t, e(t))=\Xi \int_{-\theta_{2}}^{0}\left[e^{v t} h^{T}(e(t)) h(e(t))\right. \\
& \left.-e^{\nu(t+s)} h^{T}(e(t+s)) h(e(t+s))\right] d s \\
& \leq \theta_{2} \Xi e^{v t} h^{T}(e(t)) h(e(t)) \\
& -\Xi \int_{t-\theta_{2}}^{t} e^{\nu(t+s)} h^{T}(e(t+s)) \\
& \cdot h(e(t+s)) d(t+s) \\
& \leq \theta_{2} \Xi e^{\nu t} e^{T}(t) \Lambda^{T} \Lambda e(t)-\Xi \int_{t-\rho(t)}^{t} e^{\nu s} h^{T}(e(s)) \\
& \text { - } h(e(s)) d s .
\end{aligned}
$$

It follows from Lemma 8 that

$$
\begin{aligned}
2 e^{T}(t) A(x(t)) h(e(t)) & \\
\leq & \Delta_{1} e^{T}(t) A(x(t)) A^{T}(x(t)) e(t) \\
& +\Delta_{1}^{-1} h^{T}(e(t)) h(e(t)) \\
& \leq \Delta_{1} e^{T} \stackrel{\circ}{A} \stackrel{T}{ } e(t)+\Delta_{1}^{-1} e^{T}(t) \Lambda^{T} \Lambda e(t),
\end{aligned}
$$

$$
\begin{aligned}
& 2 e^{T}(t) B(x(t-\tau(t))) z(e(t-\tau(t))) \\
& \leq \Delta_{2} e^{T} B(x(t-\tau(t))) B^{T}(x(t-\tau(t))) e(t) \\
& \quad+\Delta_{2}^{-1} z^{T}(e(t-\tau(t))) z(e(t-\tau(t))) \\
& \leq \Delta_{2} e^{T} \stackrel{\circ}{B} B^{T} e(t) \\
& \quad+\Delta_{2}^{-1} e^{T}(t-\tau(t)) \Theta^{T} \Theta e(t-\tau(t)), \\
& 2 e^{T}(t) C(x(t)) \int_{t-\rho(t)}^{t} h(e(s)) d s \\
& \leq \Delta_{3} e^{T} \dot{\circ}_{C} \dot{\circ}^{T} e(t) \\
& \quad+\Delta_{3}^{-1}\left(\int_{t-\rho(t)}^{t} h(e(t)) d s\right)^{T}\left(\int_{t-\rho(t)}^{t} h(e(t)) d s\right) .
\end{aligned}
$$

Under Lemma 17, we obtain that

$$
\begin{aligned}
& -\Xi \int_{t-\rho(t)}^{t} e^{\nu s} h^{T}(e(s)) h(e(s)) d s \\
& \leq-\frac{\nu \Xi}{e^{-\nu(t-\rho(t))}-e^{-\nu t}}\left(\int_{t-\rho(t)}^{t} h(e(s)) d s\right)^{T} \\
& \cdot\left(\int_{t-\rho(t)}^{t} h(e(s)) d s\right) \\
& \leq-\frac{\nu e^{\nu t} \Xi}{e^{\nu \rho(t)}-1}\left(\int_{t-\rho(t)}^{t} h(e(s)) d s\right)^{T} \\
& \cdot\left(\int_{t-\rho(t)}^{t} h(e(s)) d s\right) \\
& \leq-\frac{\nu e^{\nu t} \Xi}{e^{\nu \theta_{2}}-1}\left(\int_{t-\rho(t)}^{t} h(e(s)) d s\right)^{T} \\
& \cdot\left(\int_{t-\rho(t)}^{t} h(e(s))\right) .
\end{aligned}
$$

From Assumption 16, it is clear that

$$
\begin{aligned}
& \mathscr{L} V(t, e(t)) \leq e^{v t} e^{T}(t)\left[\nu-2 D+\Delta_{1} \stackrel{\circ}{A}^{T}+\Delta_{1}^{-1} \Lambda^{T} \Lambda\right. \\
& \left.+\Delta_{2} \stackrel{\circ}{B}^{T}{ }^{T}+\Delta_{3} \stackrel{\circ}{C}^{T}-2 m+\frac{\Pi e^{\gamma t}}{1-\delta}+\theta_{2} \Xi \Lambda^{T} \Lambda\right] \\
& \cdot e(t)+\Delta_{2}^{-1} e^{v t} e^{T}(t-\tau(t)) \Theta^{T} \Theta e(t-\tau(t)) \\
& +e^{v t}\left[e^{T}(t) T_{1}^{T} T_{1} e(t)\right. \\
& \left.+e^{T}(t-\tau(t)) T_{2}^{T} T_{2} e(t-\tau(t))\right]-\frac{\prod e^{\nu t}}{e^{\nu \theta_{1}}} e^{T}(t \\
& -\tau(t)) e(t-\tau(t))-\left(\Delta_{3}^{-1}-\frac{\nu e^{\nu t} \Xi}{e^{\nu \theta_{2}}-1}\right) \\
& \times\left(\int_{t-\rho(t)}^{t} h(e(s)) d s\right)^{T}\left(\int_{t-\rho(t)}^{t} h(e(s)) d s\right) .
\end{aligned}
$$


Then we have

$$
\begin{aligned}
& \mathscr{L} V(t, e(t)) \leq e^{\nu t} e^{T}(t)\left[\nu-D+\Delta_{1} \stackrel{\circ}{A}^{T}+\Delta_{1}^{-1} \Lambda^{T} \Lambda\right. \\
& +\Delta_{2} \stackrel{\circ}{B} \dot{\circ}^{T}-2 m+\Delta_{3} \stackrel{\circ}{C}^{T}+\frac{\Pi}{1-\delta}+T_{1}^{T} T_{1} \\
& \left.+\theta_{2} \Xi \Lambda^{T} \Lambda\right] e(t)+e^{\nu t} e^{T}(t-\tau(t))\left[\Delta_{2}^{-1} \Theta^{T} \Theta\right. \\
& \left.+T_{2}^{T} T_{2}-\frac{\Pi}{e^{\nu \theta_{1}}}\right] \times e(t-\tau(t))+\left(\Delta_{3}^{-1}-\frac{\nu e^{\nu t} \Xi}{e^{\nu \theta_{2}}-1}\right) \\
& \quad \times\left(\int_{t-\rho(t)}^{t} h(e(s)) d s\right)^{T}\left(\int_{t-\rho(t)}^{t} h(e(s)) d s\right) .
\end{aligned}
$$

Let

$$
\begin{aligned}
\Pi & =e^{\nu \theta_{1}}\left[\Delta_{2}^{-1} \Theta^{T} \Theta+T_{2}^{T} T_{2}\right], \\
\Xi & =\frac{e^{\nu \theta_{2}}-1}{\nu \Delta_{3} e^{\nu t}}, \\
m & =\frac{1}{2}\left(\nu-2 D+\Delta_{1} \stackrel{\circ}{A} \stackrel{A}{T}^{T}+\Delta_{1}^{-1} \Lambda^{T} \Lambda+\Delta_{2} \stackrel{\circ}{B} \dot{B}^{T}\right. \\
& \left.+\Delta_{3} \dot{\circ}^{T}+\frac{\Pi}{1-\delta}+T_{1}^{T} T_{1}+\theta_{2} \Xi \Lambda^{T} \Lambda+1\right) .
\end{aligned}
$$

Considering the condition of Corollary 14, we get

$$
\mathscr{L} V(t) \leq-e^{\vartheta t} e^{T}(t) e(t) \leq 0
$$

That is,

$$
E V(t, e(t)) \leq E V(0, e(0)), \quad \forall t \geq 0 .
$$

Combining with (52), we obtain that

$$
\begin{aligned}
E|e(t)|^{2} & =E\left|e(t)^{T} e(t)\right| \leq e^{-v t} E V(t, e(t)) \\
& \leq E V(0, e(0)) e^{-v t} .
\end{aligned}
$$

According to Definition 10, this implies that the exponential antisynchronization of systems (6) and (8) is achieved. Thus, the proof is completed.

When there is no distributed time-varying delay in system (45), the error system is introduced as follows:

$$
\begin{aligned}
& d e(t)=[-D e(t)+A(t) h(e(t)) \\
& +B(t) z(e(t-\tau(t)))+2 I+u(t)] d t \\
& +\beta(t, e(t), e(t-\tau(t))) d \omega(t) .
\end{aligned}
$$

Corollary 19. Assumptions 3, 4, and 16 hold, for a given constant $v>0$; if there exist matrix $\Pi>0$ and constant $m>0$ such that the conditions hold

$$
\begin{aligned}
\Pi & =e^{\nu \theta_{1}} T_{2}^{T} T_{2}, \\
m & =\frac{1}{2}\left(\nu-2 D+\Delta_{1} \stackrel{\circ}{ } \stackrel{\circ}{A}^{T}+\Delta_{1}^{-1} \Lambda^{T} \Lambda+\Delta_{2} \stackrel{\circ}{B}^{\circ}{ }^{T}\right. \\
& \left.+\frac{\Pi}{1-\delta}+T_{1}^{T} T_{1}+1\right),
\end{aligned}
$$

system (62) will exponentially converge to zero with the action of the delay-independent controller (48).

Proof. We construct the following Lyapunov function:

$$
\begin{aligned}
V(t, e(t))= & e^{\nu t} e^{T}(t) e(t)+\frac{1}{\mu}(M(t)-m)^{2} \\
& +\frac{\Pi}{(1-\delta)} \int_{t-\tau(t)}^{t} e^{\nu s} e^{T}(s) e(s) d s .
\end{aligned}
$$

According to the proof of Theorem 18, Corollary 19 is not difficult to obtain. Hence, it is omitted here.

Remark 20. Theorem 18 holds only when some special conditions are fulfilled: (1) all parameters meet some certain conditions; (2) the novel delay-independent adaptive controller which is much easier to implement in practice is provided. What makes that all the more remarkable is that there is no upper bound constraint on the exponent convergence speed $v$ in Theorem 18. Then we can improve the exponent convergence speed $v$ appropriately within the permissible range to achieve synchronization as soon as possible. So Theorem 18 is less conservative from a certain perspective.

\section{Numerical Simulation}

In this section, several numerical examples are offered to illustrate the effectiveness of the results obtained in the above section.

Example 21. Consider the following 2-dimensional MNNs with mixed time-varying delays:

$$
\begin{aligned}
& d x_{i}(t)=\left\{-d_{i} x_{i}(t)+\sum_{j=1}^{2} a_{i j}\left(x_{i}(t)\right) f_{j}\left(x_{j}(t)\right)\right. \\
& +\sum_{j=1}^{2} b_{i j}\left(x_{i}(t-\tau(t))\right) g_{j}\left(x_{j}(t-\tau(t))\right) \\
& \left.+\sum_{j=1}^{2} c_{i j}\left(x_{i}(t)\right) \int_{t-\rho(t)}^{t} f_{j}\left(x_{j}(s)\right) d s+I_{i}\right\} d t, \\
& i=1,2,
\end{aligned}
$$

where

$$
\begin{aligned}
& a_{11}\left(x_{1}(t)\right)= \begin{cases}0.4, & \left|x_{1}(t)\right|>\Phi_{1}, \\
0.38, & \left|x_{1}(t)\right| \leq \Phi_{1},\end{cases} \\
& a_{12}\left(x_{1}(t)\right)= \begin{cases}-1, & \left|x_{1}(t)\right|>\Phi_{1}, \\
-1.1, & \left|x_{1}(t)\right| \leq \Phi_{1},\end{cases} \\
& a_{21}\left(x_{2}(t)\right)= \begin{cases}-0.7, & \left|x_{2}(t)\right|>\Phi_{2} \\
-0.64, & \left|x_{2}(t)\right| \leq \Phi_{2}\end{cases}
\end{aligned}
$$




$$
\begin{aligned}
a_{22}\left(x_{2}(t)\right) & = \begin{cases}0.75, & \left|x_{2}(t)\right|>\Phi_{2}, \\
0.71, & \left|x_{2}(t)\right| \leq \Phi_{2},\end{cases} \\
b_{11}\left(x_{1}(t-\tau(t))\right) & = \begin{cases}-0.76, & \left|x_{1}(t-\tau(t))\right|>\Phi_{1}, \\
-0.82, & \left|x_{1}(t-\tau(t))\right| \leq \Phi_{1},\end{cases} \\
b_{12}\left(x_{1}(t-\tau(t))\right) & = \begin{cases}0.7, & \left|x_{1}(t-\tau(t))\right|>\Phi_{1}, \\
0.68, & \left|x_{1}(t-\tau(t))\right| \leq \Phi_{1},\end{cases} \\
b_{21}\left(x_{2}(t-\tau(t))\right) & = \begin{cases}0.35, & \left|x_{2}(t-\tau(t))\right|>\Phi_{2}, \\
0.29, & \left|x_{2}(t-\tau(t))\right| \leq \Phi_{2},\end{cases} \\
b_{22}\left(x_{2}(t-\tau(t))\right) & = \begin{cases}0.74, & \left|x_{2}(t-\tau(t))\right|>\Phi_{2}, \\
0.88, & \left|x_{2}(t-\tau(t))\right| \leq \Phi_{2},\end{cases} \\
c_{11}\left(x_{1}(t)\right) & = \begin{cases}0.3, & \left|x_{1}(t)\right|>\Phi_{1}, \\
0.25, & \left|x_{1}(t)\right| \leq \Phi_{1},\end{cases} \\
c_{22}\left(x_{2}(t)\right) & = \begin{cases}-0.98, & \left|x_{2}(t)\right|>\Phi_{2}, \\
-0.89, & \left|x_{2}(t)\right| \leq \Phi_{2} .\end{cases} \\
c_{21}\left(x_{2}(t)\right) & = \begin{cases}0.24, & \left|x_{1}(t)\right|>\Phi_{1}, \\
-0.21, & \left|x_{2}(t)\right|>\Phi_{2},\end{cases}
\end{aligned}
$$

For system (65), we obtain the following response system with stochastic perturbation:

$$
\begin{aligned}
& d y_{i}(t)=\left\{-d_{i} y_{i}(t)+\sum_{j=1}^{2} a_{i j}\left(y_{i}(t)\right) f_{j}\left(y_{j}(t)\right)\right. \\
& +\sum_{j=1}^{2} b_{i j}\left(y_{i}(t-\tau(t))\right) g_{j}\left(y_{j}(t-\tau(t))\right) \\
& +\sum_{j=1}^{2} c_{i j}\left(y_{i}(t)\right) \int_{t-\rho(t)}^{t} f_{j}\left(y_{j}(s)\right) d s+I_{i} \\
& \left.+u_{i}(t)\right\} d t+\beta_{i}\left(t, x_{i}(t)+y_{i}(t), x_{i}(t-\tau(t))\right. \\
& \left.+y_{i}(t-\tau(t))\right) d \omega_{i}(t),
\end{aligned}
$$

where the delay-dependent controller $u_{i}(t)$ is designed as follows:

$$
u_{i}(t)=-k_{i}(t) e_{i}(t)-m_{i}(t) e_{i}(t-\tau(t))-2 I_{i} .
$$

And the adaptive law is

$$
\begin{aligned}
& \dot{k}_{i}(t)=p_{i}\left\|e_{i}(t)\right\|^{2} e^{\nu t}, \quad k_{i}(0)=0, \\
& \dot{m}_{i}(t)=q_{i}\left\|e_{i}(t-\tau(t)) e_{i}(t)\right\| e^{\nu t}, m_{i}(0)=0, \\
& i=1,2 .
\end{aligned}
$$

Here $d_{1}=2.12, d_{2}=2.17, I_{1}=-0.01$, and $I_{2}=0.01$. The activation functions are taken as $f_{i}(s)=g_{i}(s)=\tanh (s)$, $i=1,2$. Time delays $(t)=\rho(t)=0.5 \cos (t)+0.5$. It is easy to calculate that $\theta_{1}=\theta_{2}=1, \delta=0.5$. According to Assumption 3, it can be verified that $L_{1}=L_{2}=L_{3}=1$ and $Q_{1}=Q_{2}=Q_{3}=1$. The initial conditions of system (65) are chosen as $x_{1}(t)=1.75, x_{2}(t)=0.75, t \in[-1,0]$. We take $v=1.53, p_{1}=1.61, p_{2}=1.33, q_{1}=1.23$, and $q_{2}=1.03$. And the stochastic perturbations are given such as

$$
\begin{aligned}
& \beta_{1}\left(t, e_{1}(t), e_{1}(t-\tau(t))\right) \\
& =\sqrt{0.5} e_{1}(t)+\sqrt{0.89} e_{1}(t-\tau(t)), \\
& \beta_{2}\left(t, e_{2}(t), e_{2}(t-\tau(t))\right) \\
& =-\sqrt{0.5} e_{2}(t)-\sqrt{0.56} e_{2}(t-\tau(t)) .
\end{aligned}
$$

When $\Phi_{1}=\Phi_{2}=1$, according to Theorem 11, it can be concluded that systems (65) and (67) with the above parameters achieve exponential antisynchronization in the mean square. Define error system $e_{i}(t)=y_{i}(t)+x_{i}(t)$; Figure 1 depicts the time response curves of state variables of systems (65) and (67) and the curves of synchronization errors $e_{1}(t)$ and $e_{2}(t)$ without or with the delay-dependent adaptive controller (68). It is clear that Figure 1 testifies that the synchronization errors under the effect of the delaydependent controller (68) tend to zero as time goes on.

When the time-varying delay $\tau(t)$ is not differentiable or the derivative $\dot{\tau}(t)$ is unknown or not restriction, we design the following controller:

$$
\begin{aligned}
u_{i}(t) & =-k_{i}(t)-m_{i}(t) e_{i}(t-\tau(t))-2 I_{i}, \\
\dot{k}_{i}(t) & =p_{i}\left\|e_{i}(t)\right\| e^{\nu t}, \quad k_{i}(0)=0, \\
\dot{m}_{i}(t) & =q_{i}\left\|e_{i}(t-\tau(t)) e_{i}(t)\right\| e^{\nu t}, \quad m_{i}(0)=0, \\
i & =1,2,
\end{aligned}
$$

where $\kappa_{i}=\omega_{i}=1, i=1,2$ and all other parameter values are the same as those mentioned from above.

According to Corollary 14, systems (65) and (67) under the above controller implement exponential antisynchronization. Likewise, define error system $e_{i}(t)=y_{i}(t)+x_{i}(t)$; Figure 2 shows the curves of error systems without or with controller (71). The error system with controller converges significantly to zero which is a stable state. 


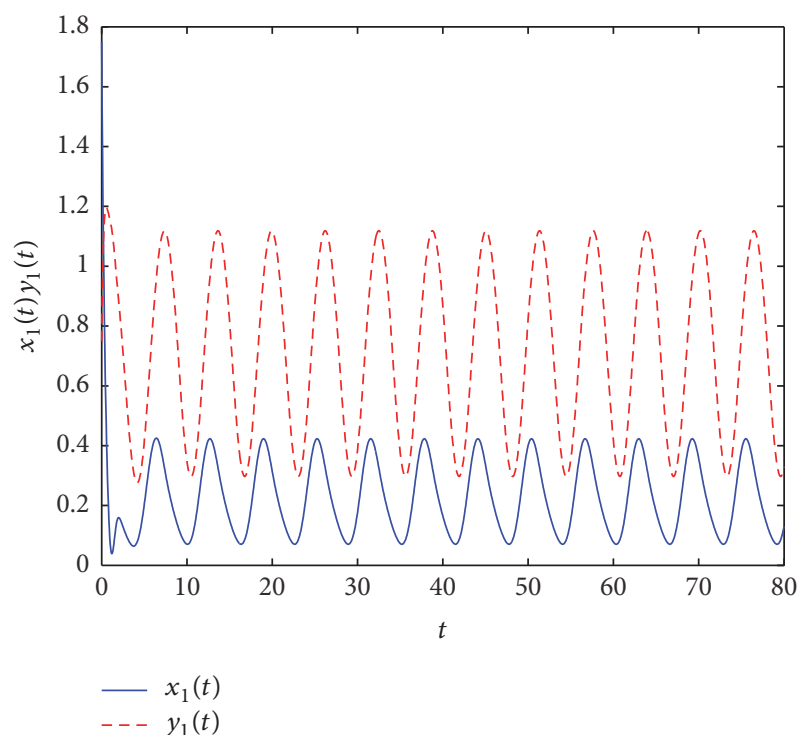

(a)

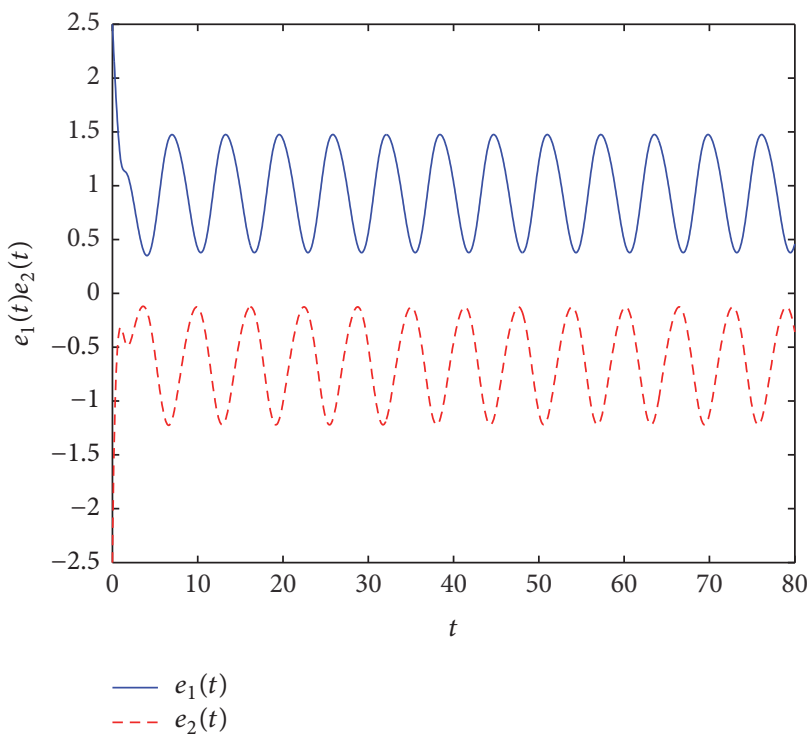

(c)

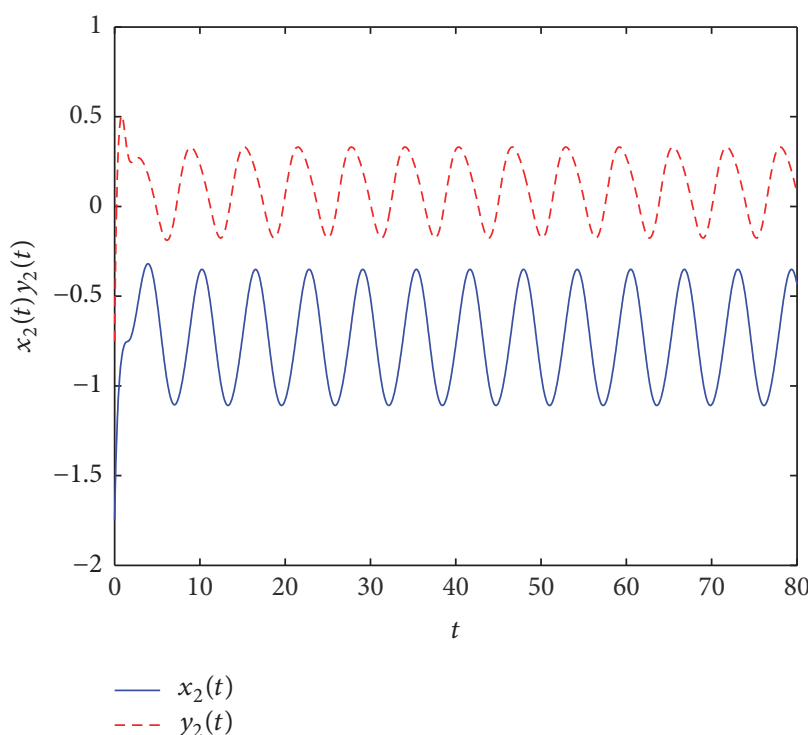

(b)

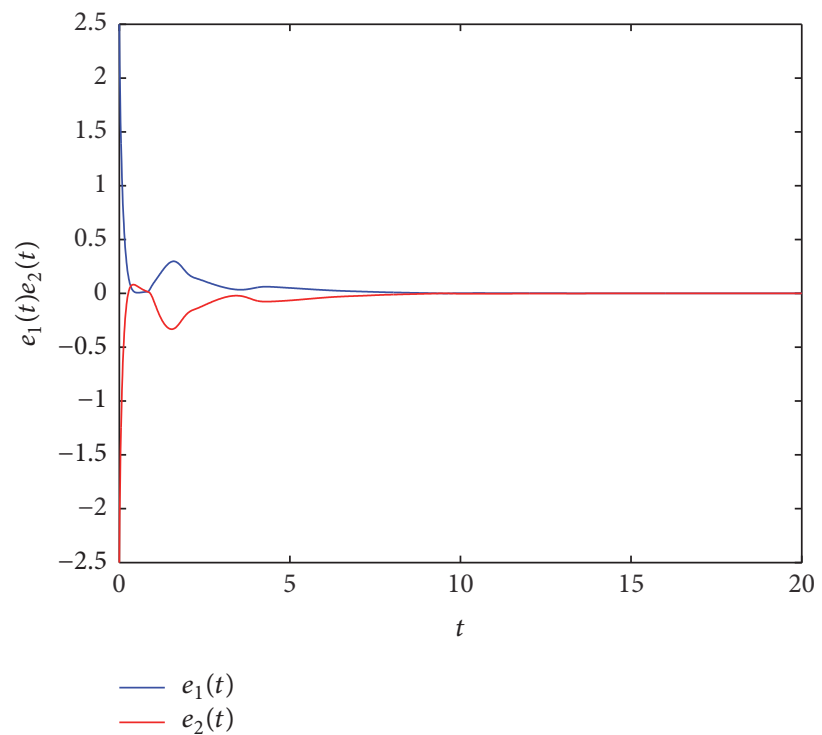

(d)

Figure 1: (a) Time response curves of states $x_{1}(t)$ and $y_{1}(t)$; (b) time response curves of states $x_{2}(t)$ and $y_{2}(t)$; (c) curves of error systems without controller; (d) curves of error systems with controller (68).

Example 22. We consider the following MNNs without stochastic perturbation as drive system; the vector form is given by

$$
\begin{gathered}
d x(t)=[-D x(t)+A(x(t)) f(x(t)) \\
+B(x(t-\tau(t))) g(x(t-\tau(t))) \\
\left.+C(x(t)) \int_{t-\rho}^{t} f(x(s)) d s+I\right] d t
\end{gathered}
$$

where

$$
A(x(t))=\left[\begin{array}{ll}
a_{11}\left(x_{1}(t)\right) & a_{12}\left(x_{1}(t)\right) \\
a_{21}\left(x_{2}(t)\right) & a_{22}\left(x_{2}(t)\right)
\end{array}\right],
$$

$$
\begin{aligned}
& B(x(t-\tau(t))) \\
& =\left[\begin{array}{ll}
b_{11}\left(x_{1}(t-\tau(t))\right) & b_{12}\left(x_{1}(t-\tau(t))\right) \\
b_{21}\left(x_{2}(t-\tau(t))\right) & b_{22}\left(x_{2}(t-\tau(t))\right)
\end{array}\right], \\
& C(x(t))=\left[\begin{array}{ll}
c_{11}\left(x_{1}(t)\right) & c_{12}\left(x_{1}(t)\right) \\
c_{21}\left(x_{2}(t)\right) & c_{22}\left(x_{2}(t)\right)
\end{array}\right], \\
& a_{11}\left(x_{1}(t)\right)= \begin{cases}0.5, & \left|x_{1}(t)\right|>\Phi_{1}, \\
0.49, & \left|x_{1}(t)\right| \leq \Phi_{1},\end{cases}
\end{aligned}
$$$$
a_{12}\left(x_{1}(t)\right)= \begin{cases}-0.6, & \left|x_{1}(t)\right|>\Phi_{1} \\ -0.67, & \left|x_{1}(t)\right| \leq \Phi_{1}\end{cases}
$$ 


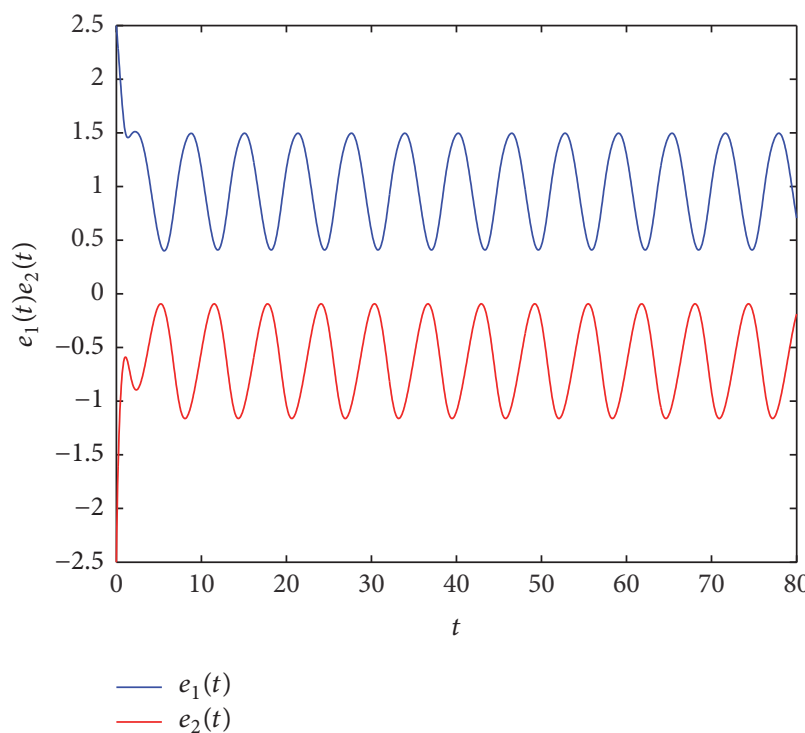

(a)

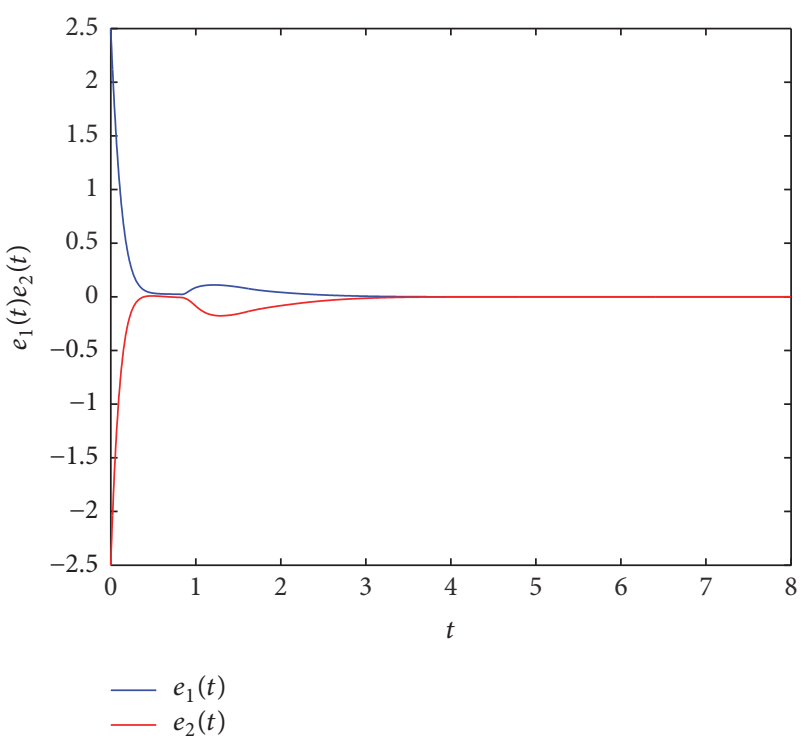

(b)

Figure 2: (a) Curves of error systems without controller; (b) curves of error systems with delay-dependent controller (71).

$a_{21}\left(x_{2}(t)\right)= \begin{cases}0.3, & \left|x_{2}(t)\right|>\Phi_{2}, \\ 0.35, & \left|x_{2}(t)\right| \leq \Phi_{2},\end{cases}$

$a_{22}\left(x_{2}(t)\right)= \begin{cases}0.4, & \left|x_{2}(t)\right|>\Phi_{2}, \\ 0.45, & \left|x_{2}(t)\right| \leq \Phi_{2},\end{cases}$

$b_{11}\left(x_{1}(t-\tau(t))\right)= \begin{cases}0.31, & \left|x_{1}(t-\tau(t))\right|>\Phi_{1}, \\ 0.29, & \left|x_{1}(t-\tau(t))\right| \leq \Phi_{1},\end{cases}$

$b_{12}\left(x_{1}(t-\tau(t))\right)= \begin{cases}-0.15, & \left|x_{1}(t-\tau(t))\right|>\Phi_{1}, \\ -0.18, & \left|x_{1}(t-\tau(t))\right| \leq \Phi_{1},\end{cases}$

$b_{21}\left(x_{2}(t-\tau(t))\right)= \begin{cases}0.83, & \left|x_{2}(t-\tau(t))\right|>\Phi_{2}, \\ 0.85, & \left|x_{2}(t-\tau(t))\right| \leq \Phi_{2},\end{cases}$

$b_{22}\left(x_{2}(t-\tau(t))\right)= \begin{cases}0.56, & \left|x_{2}(t-\tau(t))\right|>\Phi_{2}, \\ 0.58, & \left|x_{2}(t-\tau(t))\right| \leq \Phi_{2},\end{cases}$

$c_{11}\left(x_{1}(t)\right)= \begin{cases}-0.4, & \left|x_{1}(t)\right|>\Phi_{1} \\ -0.49, & \left|x_{1}(t)\right| \leq \Phi_{1}\end{cases}$

$c_{12}\left(x_{1}(t)\right)= \begin{cases}0.2, & \left|x_{1}(t)\right|>\Phi_{1} \\ 0.29, & \left|x_{1}(t)\right| \leq \Phi_{1}\end{cases}$

$c_{21}\left(x_{2}(t)\right)= \begin{cases}0.13, & \left|x_{2}(t)\right|>\Phi_{2} \\ 0.1, & \left|x_{2}(t)\right| \leq \Phi_{2}\end{cases}$

$c_{22}\left(x_{2}(t)\right)= \begin{cases}0.3, & \left|x_{2}(t)\right|>\Phi_{2}, \\ 0.33, & \left|x_{2}(t)\right| \leq \Phi_{2} .\end{cases}$
Here $D=\operatorname{diag}(1.35,1.05)^{T}, I_{1}=I_{2}=0$. The activation functions are taken as $f(s)=g(s)=\tanh (s)$. The time delays are defined as $\tau(t)=\rho(t)=0.5 \sin (t)+0.5$; then it can be verified that $\theta_{1}=\theta_{2}=1$ and $\delta=0.5$. From Assumption 3, we select $\Lambda=\Theta=\operatorname{diag}(1,1)$. The initial conditions of system (72) are chosen as $x(t)=(1.75,-1.75)^{T}, t \in[-1,0]$.

The corresponding response system under the delayindependent controller $u(t)$ is given as follows:

$$
\begin{aligned}
& d y(t)=[-D y(t)+A(x(t)) f(y(t)) \\
& +B(x(t-\tau(t))) g(y(t-\tau(t))) \\
& \left.+C(x(t)) \int_{t-\rho}^{t} f(y(s)) d s+I+u(t)\right] d t \\
& +\beta(t, e(t), e(t-\tau(t))) d \omega(t) .
\end{aligned}
$$

We choose the initial conditions as $y(t)=(0.75,-0.75)^{T}, t \in$ $[-1,0]$ and the stochastic perturbation is given as follows:

$$
\begin{aligned}
& \beta_{1}\left(t, e_{1}(t), e_{1}(t-\tau(t))\right) \\
& \quad=\sqrt{0.04} e_{1}(t)+\sqrt{0.24} e_{1}(t-\tau(t)), \\
& \beta_{2}\left(t, e_{2}(t), e_{2}(t-\tau(t))\right) \\
& \quad=\sqrt{0.04} e_{2}(t)+\sqrt{0.24} e_{2}(t-\tau(t)),
\end{aligned}
$$

where $\omega(t)=\left(\omega_{1}(t), \omega_{2}(t)\right)^{T}$ is a 2-dimensional Wiener process satisfying $E\{d \omega(t)\}=0$. From Assumption 3, it is easy to get that $T_{1}=T_{2}=\operatorname{diag}(0.08,0.48)^{T}, \Delta_{1}=\Delta_{2}=$ $\Delta_{3}=\operatorname{diag}(1,1)$. The remaining parameters of system $(74)$ are the same as system (72). 

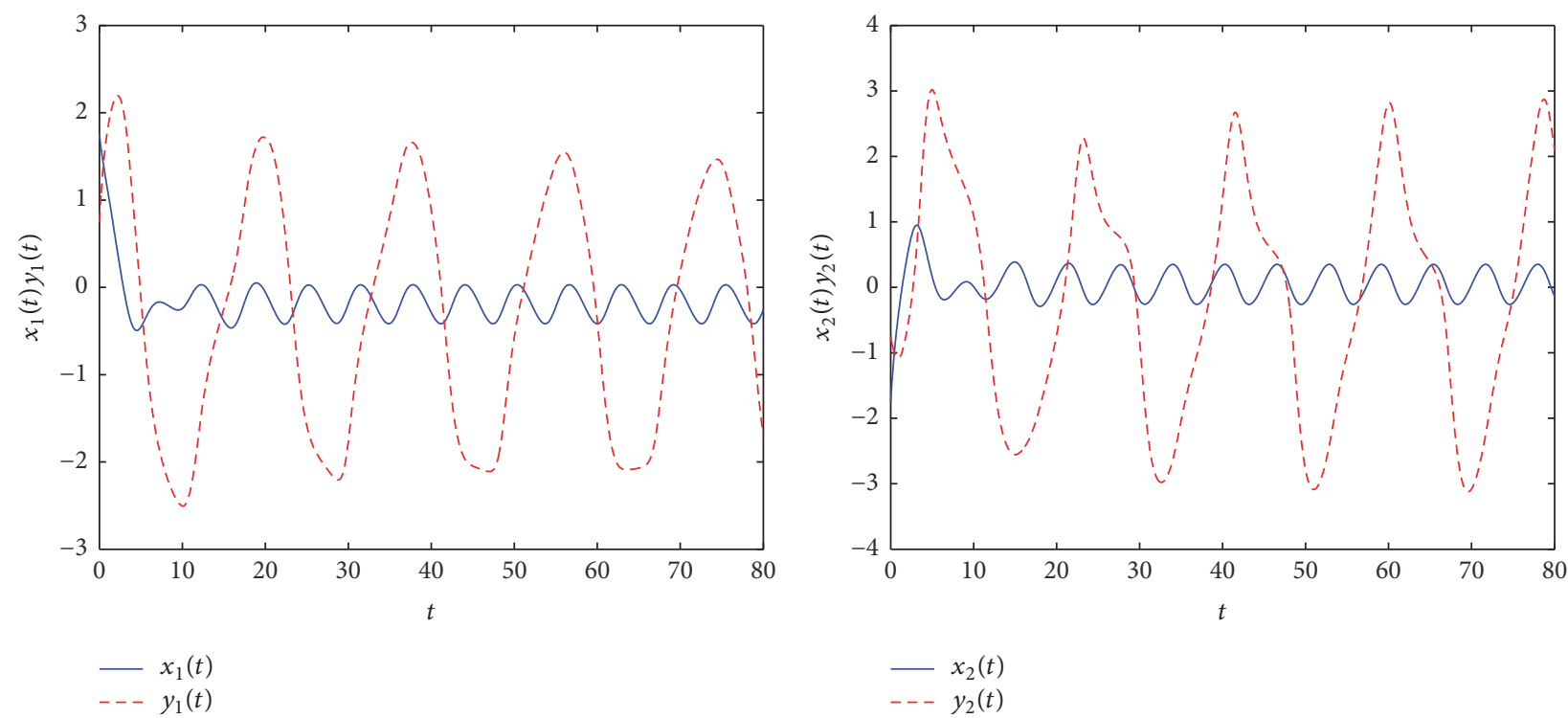

(a)
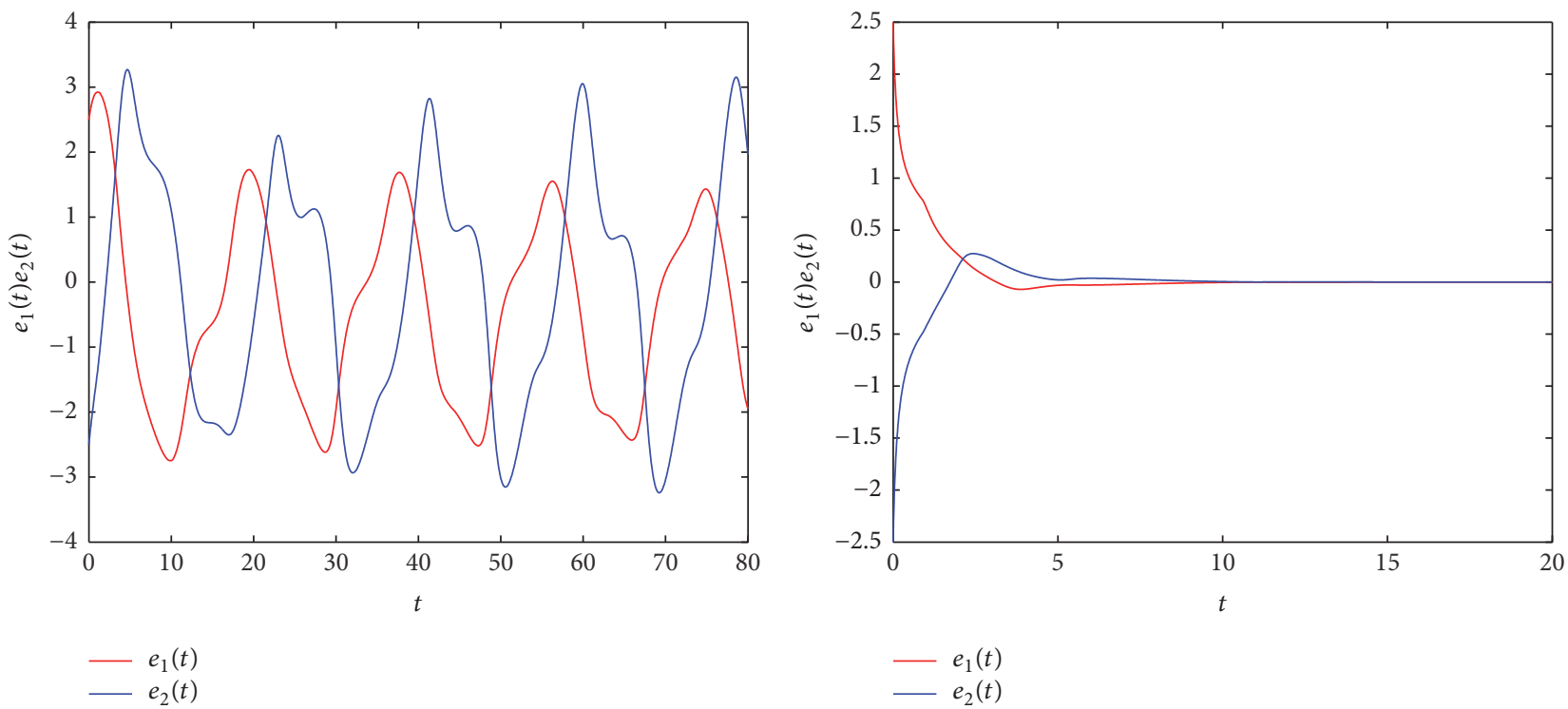

(c)

(d)

Figure 3: (a) Time response curves of states $x_{1}(t)$ and $y_{1}(t)$; (b) time response curves of states $x_{2}(t)$ and $y_{2}(t)$; (c) curves of error systems without controller; (d) curves of error systems with delay-independent controller (76).

The delay-independent controller is defined as follows:

$$
\begin{aligned}
u_{i}(t) & =-M_{i}(t) e(t)-2 I_{i}, \\
\dot{M}_{i}(t) & =\mu e^{\nu t} e^{T}(t) e(t) .
\end{aligned}
$$

In the simulation, we take $v=1.45$ and $\mu=1$. We define the error system $e(t)=y(t)+x(t)$. When $\Phi_{1}=\Phi_{2}=1$, Figure 3 depicts the time response curves of state variables of system (72) and (74) and the curves of synchronization errors $e_{1}(t)$ and $e_{2}(t)$ without or with the delay-independent adaptive controller $u(t)$. According to Theorem 18, systems (72) and (74) attain exponential antisynchronization in the mean square sense, which is verified in Figure 3.
When we ignore the impact of the distributed delay, namely, $\rho(t) \equiv 0$, system (72) will be rewritten as

$$
\begin{aligned}
\frac{d x(t)}{d t}= & -D x(t)+A(x(t)) f(x(t)) \\
& +B(x(t-\tau(t))) g(x(t-\tau(t)))+I .
\end{aligned}
$$

Then system (74) also becomes

$$
\begin{aligned}
& d y(t)=[-D y(t)+A(x(t)) h(y(t)) \\
& \quad+B(x(t-\tau(t))) z(y(t-\tau(t)))+I+u(t)] d t \\
& +\beta(t, e(t), e(t-\tau(t))) d \omega(t) .
\end{aligned}
$$




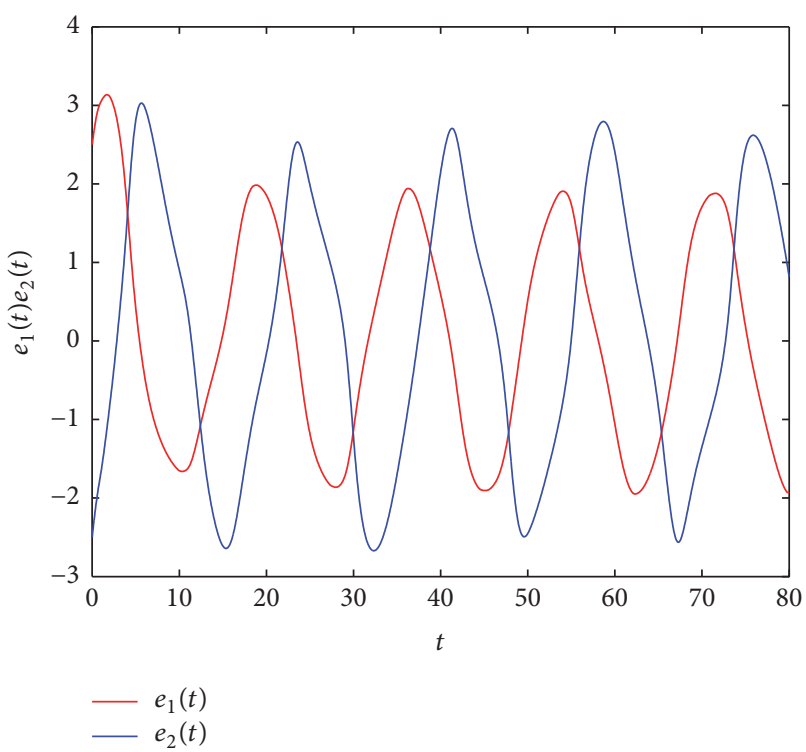

(a)

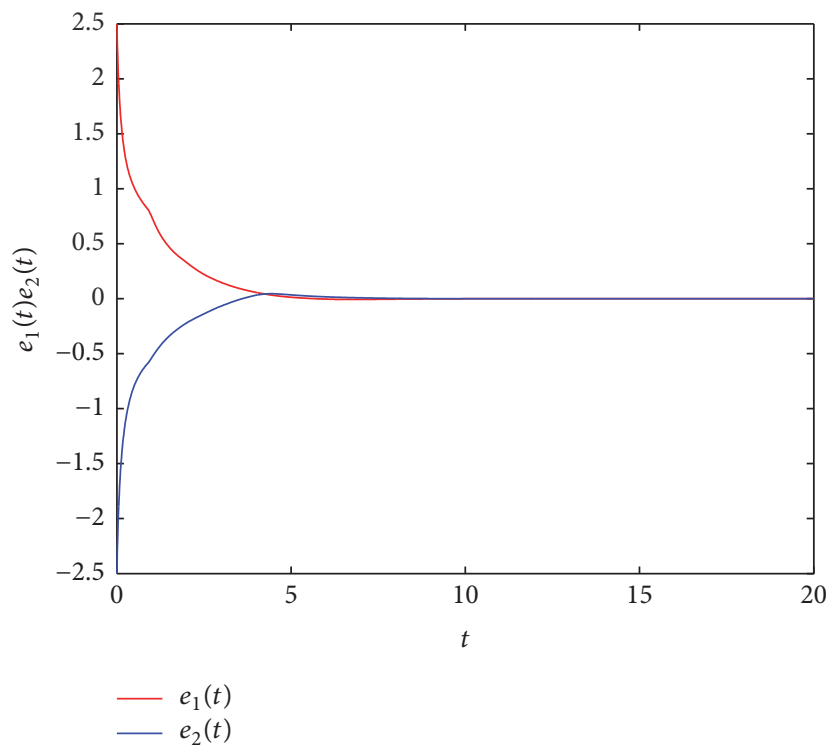

(b)

FIGURE 4: (a) Curves of error systems without controller; (b) curves of error systems with delay-independent controller (76).

Similarly, all the parameters are the same as those from above. According to Corollary 19, systems (77) and (78) under controller (76) achieve the exponential antisynchronization in the mean square sense. Define error system $e(t)=y(t)+$ $x(t)$; Figure 4 depicts the curves of error systems without or with controller (76). It is obvious that Figure 4 proves the error system converges quickly to zero.

\section{Conclusion}

In this paper, we addressed the antisynchronization issues for a class of MNNs with stochastic perturbation and mixed delays, including discrete and distributed time-varying delays. Two novel adaptive controllers were designed on the basis of delay-dependent and delay-independent to ensure that the drive system can achieve the exponential antisynchronization with the response system in spite of their initial conditions. Through the use of the Lyapunov stability method, inequality analysis technique, and the differential inclusion theory, we proposed two types of exponential antisynchronization criteria for these systems. Moreover, the obtained criteria need no excessive numerical calculation. Some numerical examples have been shown to validate the effectiveness of our theoretical results.

\section{Conflicts of Interest}

The authors declare that there are no conflicts of interest regarding the publication of this paper.

\section{Authors' Contributions}

Weiping Wang and Xiong Luo contributed equally to this work.

\section{Acknowledgments}

This work is jointly supported by the National Natural Science Foundation of China under Grants 61603032 and 61174103, the National Key Technologies R\&D Program of China under Grant 2015BAK38B01, the China Postdoctoral Science Foundation under Grant 2016M590048, the Aerospace Science Foundation of China under Grant 2014ZA74001, and the Fundamental Research Funds for the Central Universities under Grant 06500025.

\section{References}

[1] A. Wu and Z. Zeng, "Dynamic behaviors of hybrid LotkaVolterra recurrent neural networks with memristor characteristics," in Proceedings of the Annual International Joint Conference on Neural Networks (IJCNN '12), vol. 20, pp. 1-8, June 2012.

[2] S. Wen and Z. Zeng, "Dynamics analysis of a class of memristorbased recurrent networks with time-varying delays in the presence of strong external stimuli," Neural Processing Letters, vol. 35, no. 1, pp. 47-59, 2012.

[3] X. Wang, C. Li, T. Huang, and S. Duan, "Global exponential stability of a class of memristive neural networks with timevarying delays," Neural Computing and Applications, vol. 24, no. 7-8, pp. 1707-1715, 2014.

[4] Z. Guo, J. Wang, and Z. Yan, “Global exponential synchronization of two memristor-based recurrent neural networks with time delays via static or dynamic coupling," IEEE Transactions on Systems, Man, and Cybernetics: Systems, vol. 45, no. 2, pp. 235-249, 2015.

[5] X. Yang, J. Cao, and W. Yu, "Exponential synchronization of memristive Cohen-Grossberg neural networks with mixed delays," Cognitive Neurodynamics, vol. 8, no. 3, pp. 239-249, 2014.

[6] N. Li and J. Cao, "Lag synchronization of memristor-based coupled neural networks via $\omega$-measure," IEEE Transactions on 
Neural Networks and Learning Systems, vol. 27, no. 3, pp. 686697, 2016.

[7] H. Bao and J. H. Park, "Finite-time synchronization of memristor-based neural networks," in Proceedings of the 27th Chinese Control and Decision Conference (CCDC '15), pp. 17321735, IEEE, Qingdao, China, May 2015.

[8] A. Abdurahman, H. Jiang, and Z. Teng, "Finite-time synchronization for memristor-based neural networks with timevarying delays," Neural Networks, vol. 69, pp. 20-28, 2015.

[9] N. Li, J. Cao, and M. Zhou, "Anti-synchronization control for memristor-based recurrent neural networks," in Proceedings of the 11th International Symposium on Neural Networks, vol. 8866, pp. 27-34, Hong Kong and Macao, 2014.

[10] A. Wu and Z. Zeng, "Anti-synchronization control of a class of memristive recurrent neural networks," Communications in Nonlinear Science and Numerical Simulation, vol. 18, no. 2, pp. 373-385, 2013.

[11] H. Zhao, L. Li, H. Peng, J. Kurths, J. Xiao, and Y. Yang, "Anti-synchronization for stochastic memristor-based neural networks with non-modeled dynamics via adaptive control approach," European Physical Journal B, vol. 88, article no. 109, 2015.

[12] W. Zhang, J. Li, and C. Ding, "Anti-synchronization control for delayed memristor-based distributed parameter NNs with mixed boundary conditions," Advances in Difference Equations, vol. 2016, no. 1, article 320, 2016.

[13] H. Bao, J. H. Park, and J. Cao, "Matrix measure strategies for exponential synchronization and anti-synchronization of memristor-based neural networks with time-varying delays," Applied Mathematics and Computation, vol. 270, Article ID 21588, pp. 543-556, 2015.

[14] W. Wang, L. Li, H. Peng, J. Kurths, J. Xiao, and Y. Yang, "Antisynchronization control of memristive neural networks with multiple proportional delays," Neural Processing Letters, vol. 43, no. 1, pp. 269-283, 2016.

[15] W. Wang, L. Li, H. Peng et al., "Anti-synchronization of coupled memristive neutral-type neural networks with mixed timevarying delays via randomly occurring control," Nonlinear Dynamics, vol. 83, no. 4, pp. 2143-2155, 2016.

[16] Y. Song and W. Sun, "Global anti-synchronization of memristor-based recurrent neural networks with timevarying delays and impulsive effects," in Proceedings of the 6th International Conference on Intelligent Control and Information Processing (ICICIP '15), pp. 179-185, IEEE, Wuhan, China, November 2015.

[17] S. Liu and P. Liu, "Adaptive anti-synchronization of chaotic complex nonlinear systems with unknown parameters," Nonlinear Analysis. Real World Applications, vol. 12, no. 6, pp. 30463055, 2011.

[18] G. Zhang, Y. Shen, and L. Wang, "Global anti-synchronization of a class of chaotic memristive neural networks with timevarying delays," Neural Networks, vol. 46, no. 11, pp. 1-8, 2013.

[19] T. N. Lee and S. Dianat, "Stability of time-delay systems," IEEE Transactions on Automatic Control, vol. 26, no. 4, pp. 951-953, 2003.

[20] S. Wen, G. Bao, Z. Zeng, Y. Chen, and T. Huang, "Global exponential synchronization of memristor-based recurrent neural networks with time-varying delays," Neural Networks, vol. 48, pp. 195-203, 2013.

[21] G. Zhang, Y. Shen, Q. Yin, and J. Sun, "Global exponential periodicity and stability of a class of memristor-based recurrent neural networks with multiple delays," Information Sciences, vol. 232, pp. 386-396, 2013.

[22] J. Xiao, S. Zhong, and Y. Li, "New passivity criteria for memristive uncertain neural networks with leakage and time-varying delays," ISA Transactions, vol. 59, pp. 133-148, 2015.

[23] H. Wu, R. Li, S. Ding, X. Zhang, and R. Yao, "Complete periodic adaptive antisynchronization of memristor-based neural networks with mixed time-varying delays," Canadian Journal of Physics, vol. 92, no. 11, pp. 1337-1349, 2014.

[24] Z. Meng and Z. Xiang, "Passivity analysis of memristor-based recurrent neural networks with mixed time-varying delays," Neurocomputing, vol. 165, pp. 270-279, 2015.

[25] X. Yang, J. Cao, and J. Qiu, "Pth moment exponential stochastic synchronization of coupled memristor-based neural networks with mixed delays via delayed impulsive control," Neural Networks, vol. 65, pp. 80-91, 2015.

[26] Z. Meng and Z. Xiang, "Stability analysis of stochastic memristor-based recurrent neural networks with mixed timevarying delays," Neural Computing and Applications, pp. 1-13, 2016.

[27] Y. Song and S. Wen, "Synchronization control of stochastic memristor-based neural networks with mixed delays," Neurocomputing, vol. 156, pp. 121-128, 2015.

[28] H. Bao, J. H. Park, and J. Cao, "Exponential synchronization of coupled stochastic memristor-based neural networks with time-varying probabilistic delay coupling and impulsive delay," IEEE Transactions on Neural Networks and Learning Systems, vol. 27, no. 1, pp. 190-201, 2016.

[29] S. Ding, Z. Wang, Z. Huang, and H. Zhang, "Novel switching jumps dependent exponential synchronization criteria for memristor-based neural networks," Neural Processing Letters, vol. 45, no. 1, pp. 15-28, 2017.

[30] A. Chandrasekar, R. Rakkiyappan, J. Cao, and S. Lakshmanan, "Synchronization of memristor-based recurrent neural networks with two delay components based on second-order reciprocally convex approach," Neural Networks, vol. 57, pp. 7993, 2014.

[31] H. Wu, R. Li, R. Yao, and X. Zhang, "Weak, modified and function projective synchronization of chaotic memristive neural networks with time delays," Neurocomputing, vol. 149, pp. 667676, 2015.

[32] Y. Wang, L. Xie, and C. E. de Souza, "Robust control of a class of uncertain nonlinear systems," Systems and Control Letters, vol. 19, no. 2, pp. 139-149, 1992.

[33] S. Lakshmanan, J. H. Park, T. H. Lee, H. Y. Jung, and R. Rakkiyappan, "Stability criteria for BAM neural networks with leakage delays and probabilistic time-varying delays," Applied Mathematics and Computation, vol. 219, no. 17, pp. 9408-9423, 2013. 


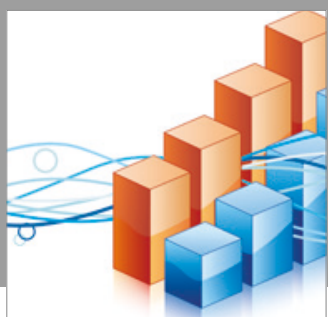

Advances in

Operations Research

vatersals

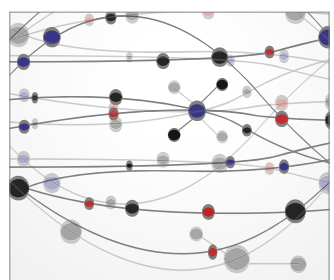

\section{The Scientific} World Journal
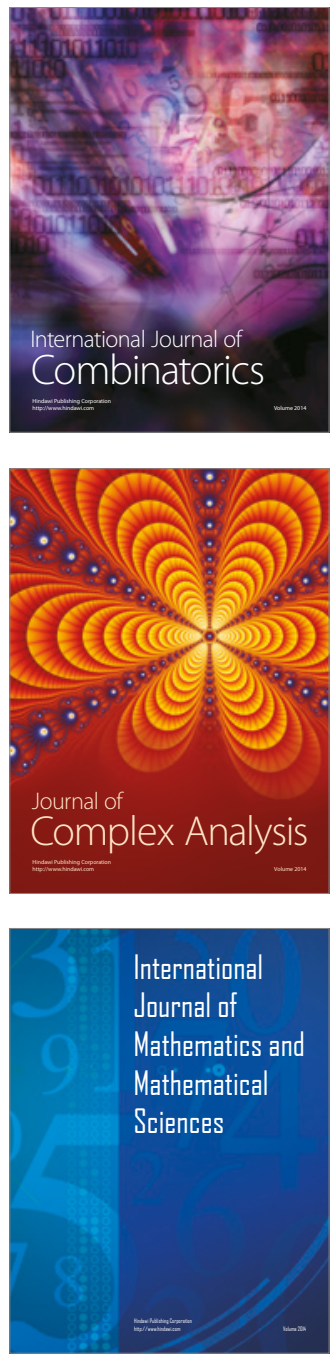
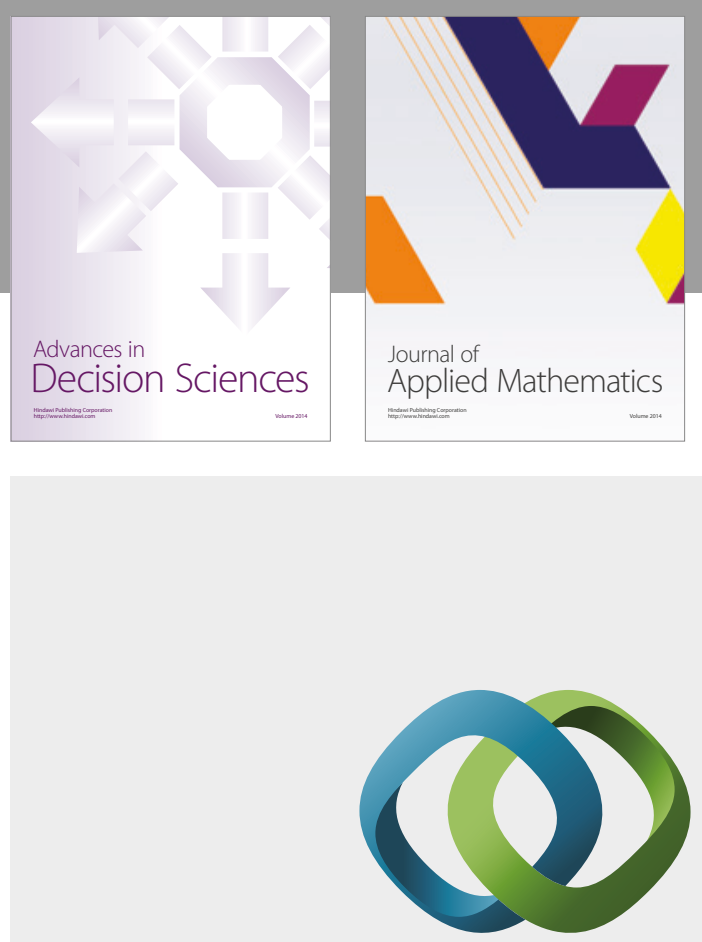

\section{Hindawi}

Submit your manuscripts at

https://www.hindawi.com
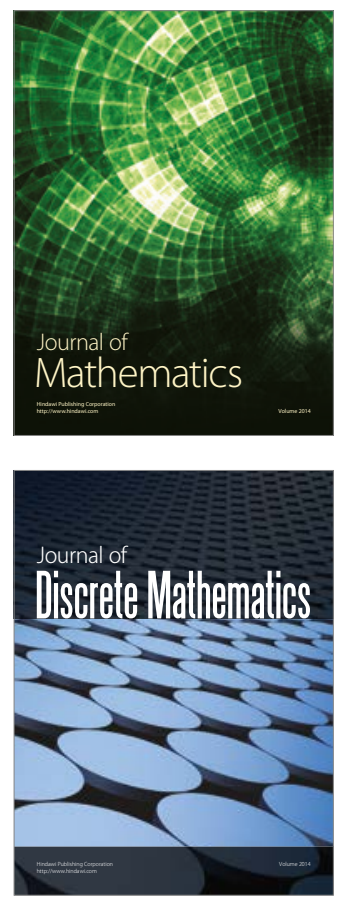

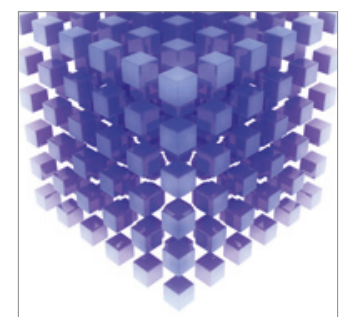

Mathematical Problems in Engineering
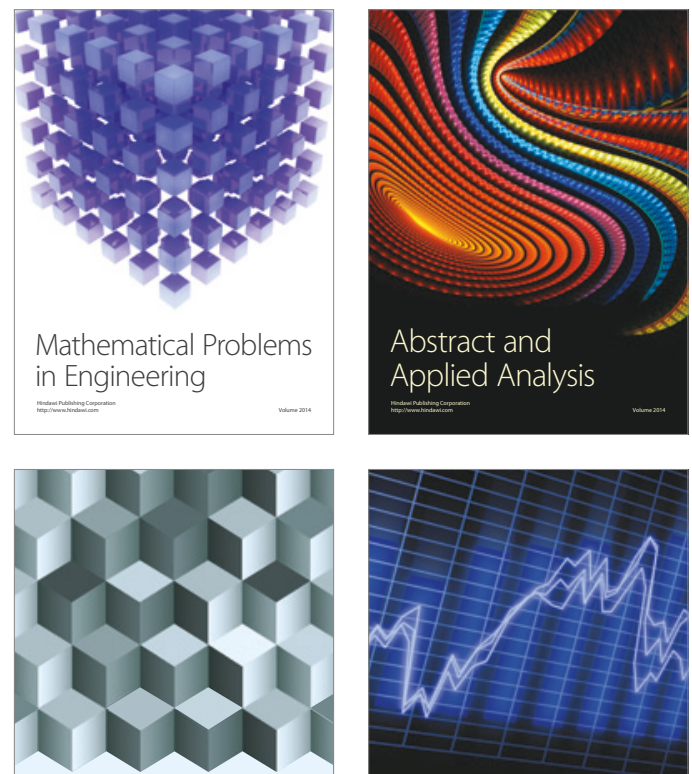

Journal of

Function Spaces

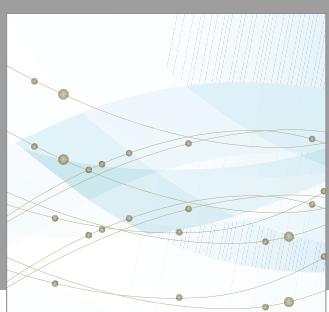

Algebra

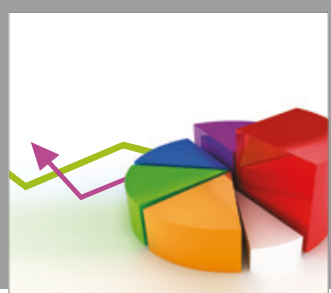

Journal of

Probability and Statistics

\section{vatemesion}

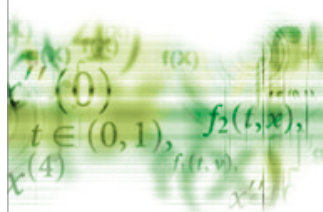

International Journal of

Differential Equations
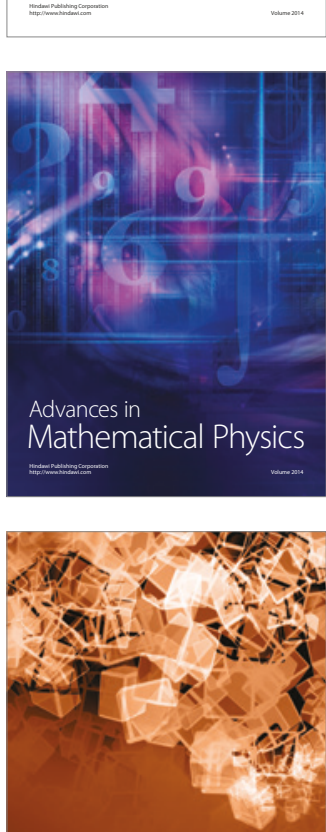

Discrete Dynamics in Nature and Society
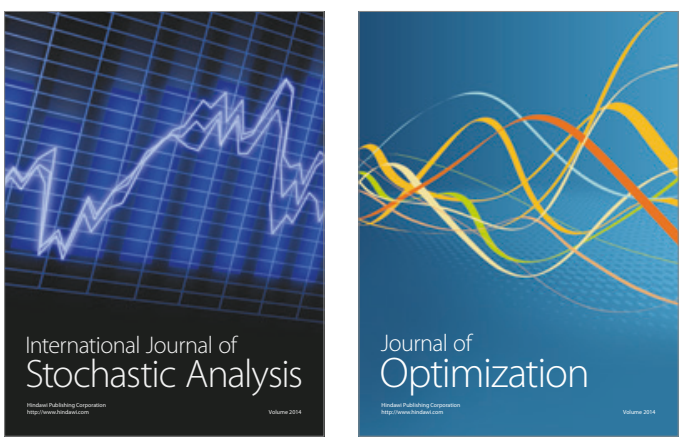